\title{
TRANSPORTATION ENERGY SURVEY DATA BOOK 1.1
}

\author{
Tatyana Gurikova \\ Macrosystems, Inc. \\ Under Subcontract No. 4000008627
}

Stacy C. Davis

Oak Ridge National Laboratory

May 2002

\author{
Prepared for \\ OAK RIDGE NATIONAL LABORATORY \\ P.O. Box 2008 \\ Oak Ridge, Tennessee 37831-6285 \\ managed by \\ UT-Battelle, LLC \\ for the \\ U.S. DEPARTMENT OF ENERGY \\ under contract DE-AC05-00OR22725
}




\section{TABLE OF CONTENTS}

Page

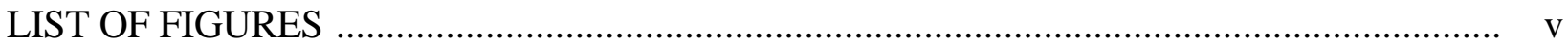

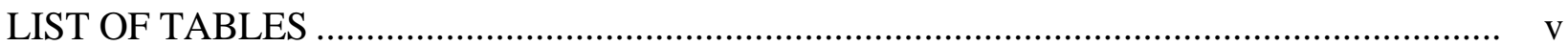

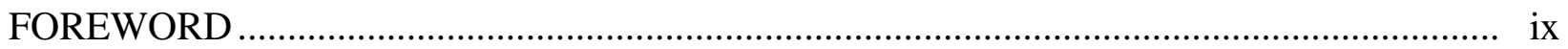

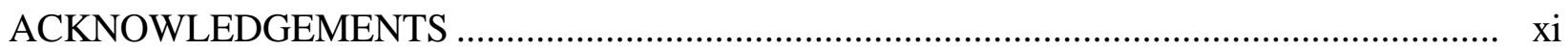

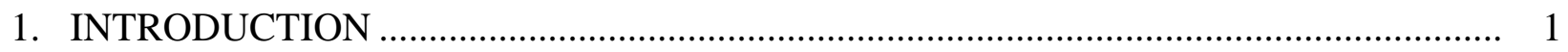

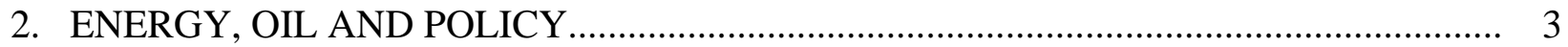

$2.1 \quad$ PUBLIC CONCERN ABOUT U.S. DEPENDENCE ON IMPORTED OIL ............... 4

2.2 PUBLIC ASSESSMENT OF THE ENERGY SITUATION ……............................... 7

2.3 PERCEIVED EFFECTS OF GASOLINE PRICES ................................................ 8

2.4 PUBLIC BELIEFS ABOUT ACTIONS TO ADDRESS THE

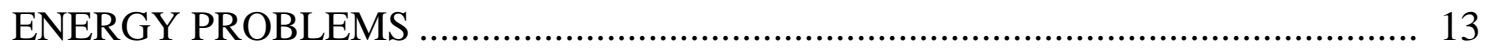

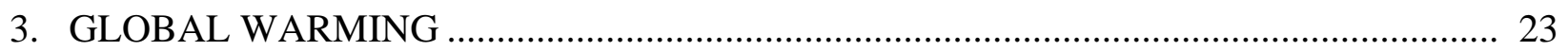

3.1 PUBLIC AWARENESS AND PERCEIVED SERIOUSNESS OF THE GLOBAL WARMING ISSUE ……………………………………………………. 24

3.2 PERCEIVED ACTIONS TO ADDRESS GLOBAL WARMING ………………...... 26

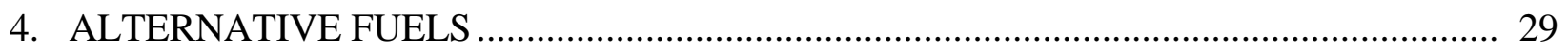

5. CONVENTIONAL, MORE FUEL-EFFICIENT, AND ADVANCED TECHNOLOGY

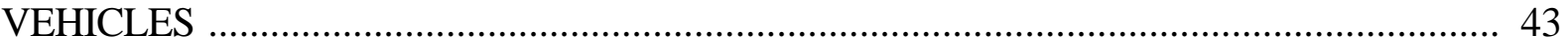

5.1 VEHICLE OWNERS' DECISIONS ABOUT CONVENTIONAL VEHICLES .......... 43

5.2 VEHICLE OWNERS' DECISIONS ABOUT MORE FUEL-EFFICIENT

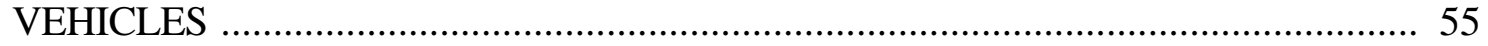

5.3 VEHICLE OWNERS' DECISIONS ABOUT ADVANCED TECHNOLOGY

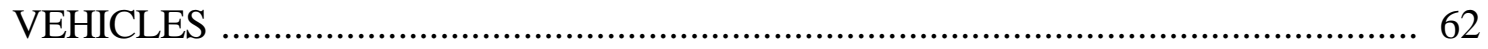

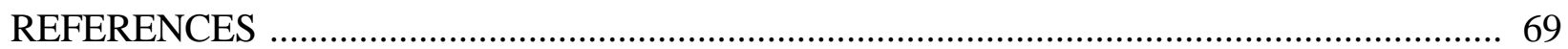




\section{LIST OF FIGURES}

Figure

Page

1 VMT and Gasoline Price Change from Same Period Previous Year 9

\section{LIST OF TABLES}

Table

Page

2.1.1 Public Perception of Aspects of U.S. Society That are Threatened by Dependence on Foreign Oil...

2.1.2 Public Approval of Statements on Dependence on Imported Oil and National Energy Policy....

2.1.3 Public Concern About the U.S. Dependence on Imported Oil ......

2.1.4 Public Perception of Imported Oil Share ....

2.2.1 Public Perception of U.S. Vulnerability to Energy Crisis

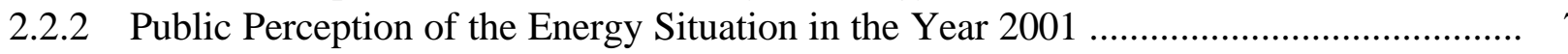

2.2.3 Public Perception of the Energy Cris is ............................................................ 8

2.3.1 Retail Unleaded Regular Gasoline Price in 2000 ............................................... 8

2.3.2 Retail Unleaded Regular Gasoline Price in 2001 ................................................. 8

2.3.3 Perceived Effects of Gasoline Price Increases on Driving - Summer 2001 ................. 9

2.3.4 Actions to Reduce Gasoline Expenditures in the Last Six Months (February-August

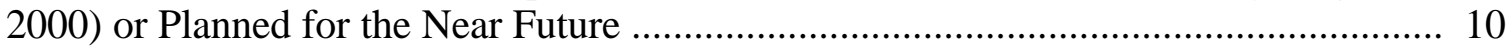

2.3.5 Perceived Effects of Recent Gasoline Price Increases................................................. 10

2.3.6 Effects of Low Gasoline Price on Driving - Winter 1999 ......................................... 11

2.3.7 Average Number of Additional Miles Driven Per Year Due to Low Gasoline Prices -

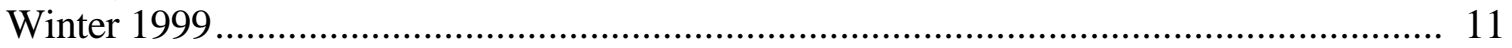

2.3.8 Public Assessment of Nature of Current Rise in Gasoline Prices ............................... 12

2.3.9 Public Perception of Gas Prices One to Six Months from Today................................ 12

2.3.10 Public Concern About the Price of Gasoline in 2002 ................................................. 13

2.4.1 Public Preference for Solving the Nation's Energy Problems ..................................... 13

2.4.2 Public Perception of Actions That Should Be Taken by Individuals, Government, and/or Business to Reduce Dependence on Imported Oil

2.4.3 Actions Taken to Reduce Oil Dependence in the Last Six Months (February-August 2000) or Planned to Be Taken in the Near Future ................................................. 15

2.4.4 Public Preference for Saving One Gallon of Fuel per Week ...................................... 16

2.4.5 Possible Actions Taken by Individuals to Reduce Fuel Consumption.......................... 17

2.4.6 Public Perception of Ways to Deal with the Energy Situation (Gallup Poll) ................. 18

2.4.7 Public Perception of Ways to Deal with the Energy Situation (NBC/WSJ) ................... 19

2.4.8 Public Support of Federal Government Actions for U.S. Energy Needs ....................... 20

2.4.9 Public Perception of Federal Government's Highest Priority ................................... 20

2.4.10 Policies the Public Would Most/Least Support to Reduce Dependence on Imported Oil 21

3.1.1 Level of Public Awareness of Global Warming .......................................................... 24

3.1.2 Level of Public Awareness of Kyoto Conference....................................................... 24

3.1.3 Perceived Seriousness of Global Warming Threat ................................................. 25 
3.1.4 Public Ranking of Most Important Transportation Problem for the United States in the Year 2020 ..... 25

3.2.1 Public Support for Actions to Address Global Warming ............................................. 26

3.2.2 Public Preference for Policy to Reduce Greenhouse Gas Emissions from Vehicles....... 26

3.2.3 Preferred Strategies for Combating Global Warming ................................................. 27

3.2.4 Perceived Contribution by Less-Developed Countries Toward Production of Greenhouse Gas Emissions

3.2.5 Public Perception of Actions Which Should Be Required of Less-Developed Countries to Reduce Greenhouse Gas Emissions.

4.1.1 Public Perception of Year in Future When Gasoline and Diesel Will Become Too

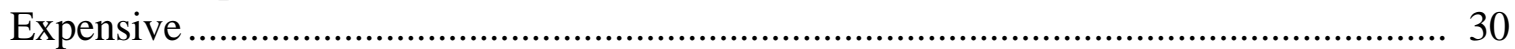

4.1.2 Public Perception of Which Fuel Will Replace Gasoline and Diesel ............................ 30

4.1.3 Public Perception of Best Fuel for Use in Personal Vehicles When Gasoline Is No Longer Available

4.1.4 Reasons Electricity Would Be the Best Fuel for Use in Personal Vehicles When Gasoline Is No Longer Available

4.1.5 Reasons Ethanol Would Be the Best Fuel for Use in Personal Vehicles When Gasoline Is No Longer Available

4.1.6 Reasons Hydrogen Would Be the Best Fuel for Use in Personal Vehicles When Gasoline Is No Longer Available

4.1.7 Public Perception of Worst Fuel for Use in Personal Vehicles When Gasoline Is No Longer Available

4.1.8 Reasons Ethanol Would Be the Worst Fuel for Use in Personal Vehicles When Gasoline Is No Longer Available

4.1.9 Reasons Hydrogen Would Be the Worst Fuel for Use in Personal Vehicles When Gasoline Is No Longer Available

4.1.10 Reasons Electricity Would Be the Worst Fuel for Use in Personal Vehicles When Gasoline Is No Longer Available

5.1.1 Total Number of Years Individuals Plan to Keep Their Vehicle ..................................... 44

5.1.2 Vehicle Acquisition Data ........................................................................................... 45

5.1.3 Primary Reason for Acquiring Current Vehicle ………............................................... 45

5.1.4 Trends in Vehicle Attribute Preference, Selected Yesars 1980-2001 ............................ 46

5.1.5 Public Perception of Vehicle Size vs. Safety.............................................................. 47

5.1.6 Towing Package Preference for a New Vehicle .......................................................... 48

5.1.7 Expected Off-Road Use of a New Vehicle ………..................................................... 49

5.1.8 Expected Expenditure for a New Vehicle ………....................................................... 50

5.1.9 Major Reasons for Preferring a Car to Other Vehicle Types........................................... 51

5.1.10 Current Vehicle Replacement Methods ................................................................... 53

5.1.11 Public Preference for Purchasing New or Used Vehicles............................................... 54

5.1.12 Public Perception of the Effect of New Vehicle Price Increases on Purchasing Decisions 
5.2.1 Location of Fuel Economy Data Used for Last Vehicle Purchase ............................... 56

5.2.2 Public Interest for Additional Information on the Environmental Impacts of New

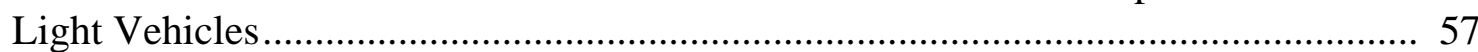

5.2.3 Public Preference Toward Purchase of Cars with Different Weight, Acceleration and Fuel Economy Parameters ................................................................................. 58

5.2.4 Public Perception of Motivation Required to Purchase a More Fuel Efficient Vehicle

5.2.5 Additional Amount the Public is Willing to Pay Extra for Vehicle With a 10 Percent Increase in Fuel Economy.....

5.2.6 Public Preference Toward Purchasing a More FuelEfficient Engine with Different Fuel Availability Options

5.2.7 Site of Review of the Government's Fuel Economy Guide ........................................ 61

5.3.1 Amount of Information Heard Pertaining to Advanced Hybrid-Electric Powertrains . 62

5.3.2 Names of Advanced Hybrid-Electric Vehicles Known by the Public 63

5.3.3 Public's Willingness to Consider the Purchase of a Diesel Engine With a 40 Percent Increase in Fuel Economy and Additional Costs of $\$ 1,500$.

5.3.4 Additional Amount the Public is Willing to Pay for a Diesel Engine Getting 30 Miles per Gallon Compared to a Gasoline Engine Getting 20 Miles per Gallon .................... 65

5.3.5 Purchase Preference Between Diesel and Gasoline Vehicles by Vehicle Type ........... 66

5.3.6 Reasons for Choosing a Diesel Option...................................................................... 67

5.3.7 Reasons for Rejecting a Diesel Option.................................................................. 68 


\section{FOREWORD}

This report has been assembled to provide the Office of Transportation Technologies (OTT) with an idea of how the American public views various transportation and environmental issues.

Some of the findings are presented below to illustrate the type of information in the report.

This is how respondents have answered some of the questions in the report:

1. $86 \%$ strongly or somewhat agree that decreasing our dependence on foreign oil is important to our national security. [11/01, Table 2.1.2]

2. $86 \%$ are very or somewhat concerned the U.S. is dependent on imported oil. [4/01, Table 2.1.3]

3. Strong support for mandating more fuel efficient new vehicles:

a. $85 \%$ [5/01, Table 2.4.7]

b. $87 \%$ [6/01, Table 2.4.8]

c. $89 \%$ [4/01, Table 2.4.9]

4. But, if more efficient vehicles cost more, about a third of the respondents unwilling to pay more for higher efficiency [11/01, Table 5.2.5]

5. If we had to reduce dependence on imported oil using four options,

a. $48 \%$ favor making personal vehicles more efficient

b. $24 \%$ favor tax refunds for higher efficiency vehicles

c. $11 \%$ favor higher taxes on less efficient vehicles

d. $6 \%$ favor higher taxes on gasoline and diesel fuel

e. $11 \%$ had no answer [5/01, Table 2.4.11]

6. When ranking the most important problem (of the five provided) for the U.S. in the year 2020:

a. $34 \%$ chose traffic congestion

b. $28 \%$ chose availability and/or price of gasoline

c. $14 \%$ chose global warming

d. $12 \%$ chose local air pollution

e. $7 \%$ chose traffic deaths and injuries

f. $4 \%$ had no answer [12/00, Table 3.1.4]

7. When provided with two options to reduce transportation greenhouse gases (that would have about equal overall costs),

a. $70 \%$ chose $3 \%$ tax for new vehicles

b. $17 \%$ chose 25 -cent tax per gallon of gasoline

c. $13 \%$ had no answer [2/98, Table 3.2.2]

8. What fuel will replace gasoline and diesel when they become too expensive?

a. $33 \%$ said electricity

b. $12 \%$ said solar

c. $11 \%$ said ethanol or methanol

d. $6 \%$ said natural gas

e. $3 \%$ said hydrogen

f. $35 \%$ said other fuels or had no answer [8/98, Table 4.1.2]

9. Of the three options, which would be best (or worst) to replace gasoline :

a. $52 \%$ said electricity was best, $15 \%$ said it would be the worst choice

b. $21 \%$ said ethanol was best, $28 \%$ said it would be the worst choice 
c. $15 \%$ said hydrogen was best, $27 \%$ said it would be the worst choice $[12 / 00$, Tables 4.1.3 and 4.1.7]

10. When fuel economy is provided as one choice to be rated as the most important attribute in the choice of a new vehicle, it was selected by $42 \%$ of the respondents in 1980 (when gasoline prices were very high). It was chose by only $4 \%$ of the respondents in 1987 and 1988. It rose to 10\% in 2000 and 2001. [Various years, Table 5.1.5]

11. Have seen a copy of the government's Fuel Economy Guide:

a. $16 \%$ in a showroom

b. $2 \%$ on the Internet

c. $6 \%$ anywhere else [2/99, Table 5.2.7]

12. Able to name a hybrid vehicle for sale in the U.S.

a. Honda $15 \%$ [8/00] and $24 \%$ [11/01]

b. Toyota 4\% [8/00] and 11\% [11/01, Tables 5.3.1 and 5.3.4]

The issue that might need the most attention from OTT is the finding that the public tends to have a fairly negative and ill-informed attitude concerning ethanol and hydrogen, two fuels that hold promise for the future. 


\section{ACKNOWLEDGEMENTS}

I would like to thank Philip Patterson of the U.S. Department of Energy and Margaret Singh of Argonne National Laboratory for providing comments and suggestions on improving this report. In addition, I thank Alicia Birky for providing survey data on global warming. 


\section{INTRODUCTION}

The transportation sector is the major consumer of oil in the United States. In 2000, the transportation sector's share of U.S. oil consumption was 68 percent (U.S. DOE/EIA, 2001a, Table 2.5, p. 33, Table 1.4, p.7). As a result, the transportation sector is one of the major producers of greenhouse gases. In 2000, the transportation sector accounted for one-third (33 percent) of carbon emissions (U.S. DOE/EIA, 2000b, Table 5, p.28). In comparison, the industrial sector accounted for 32 percent and residential and commercial sector for 35 percent of carbon emissions in 2000. Carbon emissions, together with other gases, constitute greenhouse gases that are believed to cause global warming. Because that the transportation sector is a major oil consumer and producer of greenhouse gases, the work of the Analytic Team of the Office of Transportation Technologie s (OTT) focuses on two main objectives: (1) reduction of U.S. oil dependence and (2) reduction of carbon emissions from vehicles.

There are two major factors that contribute to the problem of U.S. oil dependence. First, compared to the rest of the world, the United States does not have a large oil reserve. The United States accounts for only 9 percent of oil production (U.S. DOE/EIA, 2001c, Table 4.1C). In comparison, the Organization for Petroleum Exporting Countries (OPEC) produces 42 percent of oil, and the Persian Gulf accounts for 28 percent. (U.S. DOE/EIA, 2001c, Table 1.1A). More than half (54 percent) of oil consumed in the United States is imported (U.S. DOE/EIA, 2001a, Table 1.8, p. 15). Second, it is estimated that the world is approaching the point at which half of the total resources of conventional oil believed to exist on earth will have been used up (Birky et. al., 2001, p. 2).

Given that the United States is highly dependent on imported oil and that half of the world's conventional oil reserves will have been used up in the near future, the OTT's goal is to ensure an adequate supply of fuel for vehicles. There are three ways to achieve this goal: efficiency, substitution, or less travel. A reduction in oil usage will result in a reduction of carbon emissions.

Successful transition to alternative types of fuel and advanced technology vehicles may depend on awareness of U.S. dependence on imported oil and the U.S. energy situation. Successful transition may also depend on knowledge of alternative types of fuels and advanced technologies. The Transportation Energy Survey Data Book 1.1 examines the public's knowledge, beliefs and expectations of the energy situation in the United States and transportation energy-related issues.

The data presented in the report have been drawn from multiple sources: surveys conducted by the Opinion Research Corporation International (ORCI) for National Renewable Energy Laboratory (NREL) that are commissioned and funded by OTT, Gallup polls, ABC News/Washington Post polls, NBC News/Wall Street Journal polls, polls conducted by the Ipsos-Reid Corporation, as well articles from The Washington Post (2001) and other sources. All surveys are telephone interviews conducted with randomly selected national samples of adults 18 years of age and older. Almost all surveys were conducted before the September 11, 2001 terrorist attacks, with the only exceptions being the November 2001 ORCI survey and the November 2001 survey conducted by the Ipsos-Reid Corporation. 
The Transportation Energy Survey Data Book 1.1 consists of four sections. Section 2 examines public concern about U.S. dependence on imported oil, public assessment of the energy situation in the United States, and perceived effects of gasoline prices on individuals and households. In addition, this section focuses on public expectations and Federal Government actions that can be undertaken to deal with the energy situation and reduce dependence on imported oil.

Section 3 examines public awareness of global warming issues and perceived strategies of the United States and other lesser-developed countries to combat global warming. Section 4 analyzes what Americans think about alternative fuels such as electricity, ethanol, hydrogen and other fuel types.

Section 5 focuses on conventional and advanced technology vehicles. In this report, advanced technology vehicles include hybrid electric and diesel vehicles. The section examines the decisions vehicle owners make about their vehicles, as well as decisions about replacing vehicles. Section 5 also analyzes owners' decisions about purchasing more fuetefficient vehicles and advanced technology vehicles.

Most of the tables presented in the report are the results of survey questions that required respondents to choose among specific answers. When this type of question is asked, the interviewer rotates the order in which the choices are given in order to reduce bias. Some survey questions allowed the respondent to provide any response; these questions are referred to as open-ended questions. Some tables present results of questions in which respondents are allowed to provide more than one answer. Because of a possibility of multiple responses to the same question, results of these tables will not sum to 100 percent.

The Transportation Energy Survey Data Book 1.1 is a continuation of a joint effort of the OTT analytic team. It builds upon the Data Book on Vehicle Consumer Characteristics and Trends that started in 1997 as a working report to inform OTT project managers of important vehicle market characteristics to ensure that OTT-supported technologies meet the needs and desires of consumers. 


\section{ENERGY, OIL AND POLICY}

Public opinion polls reveal that the U.S. public perceives the country's oil dependence as a serious threat to jobs and economy, the U.S. standard of living, national security and the environment (Table 2.1.1). After the terrorist attacks on September 11, 2001, an overwhelming majority of Americans believe that decreasing U.S. dependence on foreign oil is important to national security (Table 2.1.2). Because of this perception, U.S. adults are concerned about dependence on imported oil (Table 2.1.3), but many do not have an accurate idea of how much oil is imported (Table 2.1.4).

Concern about oil dependence leads to the public's assessment of the energy situation in the country as serious (Table 2.2.1). Americans are likely to change their evaluation of the seriousness of the energy situation based on recent events. U.S. adults are more likely to evaluate the United States as not just vulnerable to, but heading into and already in, an energy crisis as the economic situation deteriorates over time (Tables 2.2.2, 2.2.3).

U.S. dependence on imported oil manifests itself in volatile gasoline prices. A number of surveys researched the U.S. public's driving expectations when gasoline prices have gone up and down. When gasoline prices go up, a majority of the adult population tend to say it would not reduce the amount of driving because of the increase (Table 2.3.3). However, when asked later whether they actually drove or planned on driving as much as they did when gasoline prices were lower, Americans reported a decrease in the amount of their driving (Table 2.3.4). This finding is consistent with the graph shown in Figure 1. The graph illustrates that Americans actually drive less when gasoline prices increase. A majority of U.S. adults report that gasoline price increases have not caused financial hardship for them or their households (Table 2.3.5). (Only the May 2001 Gallup survey revealed that almost one out of two Americans has suffered financial hardship because of gasoline price increase.) However, when asked about actions they undertook or planned to undertake in the near future to reduce gasoline expenditures, an overwhelming majority of Americans reported a change in lifestyle due to a gasoline price increase (Table 2.3.4).

When gasoline prices go down, the U.S. public is not likely to report that they would drive more because of the decrease (Table 2.3.6). However, the fact is that they do drive more when gasoline prices are low (Table 2.3.7). This finding is consistent with the relationship between vehicle miles traveled (VMT) and gasoline price shown in Figure 1.

Surveys have revealed that U.S. adults are likely to change their views on the nature of gasoline price changes based on their assessment of the seriousness of the energy situation in the country (Table 2.3.8). According to surveys, Americans are likely to evaluate gas price increases as a more permanent change than temporary fluctuations in prices when they evaluate the energy situation in the country as heading into, or already in, an energy crisis. When they think of gasoline price increases as of a more permanent change than temporary fluctuations in prices, Americans are more likely to believe that prices will go up in the future (Table 2.3.9). In addition, U.S. adults are likely to be concerned about gasoline prices in the future when they believe price increases are of a more permanent nature (Table 2.3.10).

In order to reduce U.S. oil dependence and deal with the energy situation, the U.S. public favors energy conservation over energy production (Table 2.4.1, 1.4.7, 1.4.8). One out four U.S. adults 
said he/ she purchased or planned on purchasing a more fuel-efficient vehicle as an action to reduce oil dependence (Table 2.4.2). Besides that, they mentioned reduction of the amount of driving and greater use of mass transit and carpool as a way of saving fuel (Tables 2.4.3, 2.4.4).

A number of surveys addressed mandatory improvements of vehicle fuel economy. According to these surveys, the U.S public showed strong support of a requirement for automakers to improve vehicle fuel efficiency. Mandates on more fuelefficient cars received consistent support in all surveys. (Tables 2.4.5 - 2.4.7). A survey that asked people to compare four different policies on oil dependence reduction found that a regulation to make personal vehicles more efficient received most support. This policy received much higher approval than higher fuel taxes or less-efficient vehicles (Table 2.4.9). However, none of the surveys discussed the fuel economy level that should be mandated.

\subsection{PUBLIC CONCERN ABOUT U.S. DEPENDENCE ON IMPORTED OIL}

Q2.1.1: Some people believe that depending on this much foreign oil threatens various aspects of our society while others do not believe depending on this much foreign oil threatens us in any way. Please tell me how serious a threat you think our dependence on foreign oil is to each of the following: very serious, somewhat serious, not too serious, or not at all serious.
A. Our national security
B. Jobs and economy
C. The environment
D. Our standard of living

Table 2.1.1. Public Perception of Aspects of U.S. Society That Are Threatened by Dependence on Foreign Oil

\begin{tabular}{lcc}
\hline $\begin{array}{l}\text { Aspects of Society to which Dependence on } \\
\text { Foreign Oil Represents a "Very Serious" }\end{array}$ & Number & Percent \\
or "Somewhat Serious" Threat & & \\
\hline Jobs and the economy & 858 & 86 \\
U.S. standard of living & 828 & 83 \\
U.S. national security & 769 & 77 \\
The environment & 702 & 70 \\
\hline
\end{tabular}

Source: Research/Strategy/Management, Inc., October 21, 1998, N=1,003. 
Q2.1.2: Do you strongly agree, somewhat agree, somewhat disagree, or strongly agree with the following statements?

Table 2.1.2. Public Approval of Statements on Dependence on Imported Oil and National Energy Policy

\begin{tabular}{|c|c|c|c|c|c|c|}
\hline Statements & $\begin{array}{l}\text { Strongly } \\
\text { Agree } \\
(\%) \\
\end{array}$ & $\begin{array}{c}\text { Some- } \\
\text { what } \\
\text { Agree } \\
(\%)\end{array}$ & $\begin{array}{c}\text { Some- } \\
\text { what } \\
\text { Disagree } \\
(\%) \\
\end{array}$ & $\begin{array}{c}\text { Strongly } \\
\text { Disagree } \\
(\%) \\
\end{array}$ & $\begin{array}{l}\text { No } \\
\text { Opinion } \\
(\%) \\
\end{array}$ & $\begin{array}{c}\text { Total } \\
(\%)\end{array}$ \\
\hline $\begin{array}{l}\text { Decreasing our dependence } \\
\text { on foreign oil and gas is } \\
\text { important to our national } \\
\text { security. }\end{array}$ & 49 & 37 & 7 & 5 & 2 & 100 \\
\hline $\begin{array}{l}\text { Increasing domestic } \\
\text { production of oil, gas is } \\
\text { important to our national } \\
\text { security. }\end{array}$ & 38 & 40 & 10 & 8 & 4 & 100 \\
\hline $\begin{array}{l}\text { New technologies have made } \\
\text { it possible to explore for oil } \\
\text { and gas in environmentally } \\
\text { friendly ways. }\end{array}$ & 29 & 46 & 12 & 5 & 8 & 100 \\
\hline $\begin{array}{l}\text { The introduction of a } \\
\text { national energy policy will } \\
\text { help to boost energy } \\
\text { conservation efforts. }\end{array}$ & 18 & 54 & 15 & 6 & 7 & 100 \\
\hline $\begin{array}{l}\text { Passing a national energy } \\
\text { policy will improve the } \\
\text { economy and put people } \\
\text { back to work. }\end{array}$ & 19 & 45 & 21 & 7 & 8 & 100 \\
\hline
\end{tabular}

Source : Ipsos-Reid Inc., November 14, 2001, N=532

${ }^{1}$ Half sample 
Q2.1.3: The United States now imports 54 percent of its oil supplies. This fraction is growing. How concerned are you about the fact that the United States is dependent on imported oil?

Table 2.1.3. Public Concern About U.S. Dependence on Imported Oil

\begin{tabular}{lrr}
\hline Degree of Public Concern & Number & Percent \\
\hline Very concerned & 472 & 50 \\
Somewhat concerned & 343 & 36 \\
Not at all concerned & 120 & 13 \\
Don't know & 6 & 1 \\
Total & 941 & 100 \\
\hline
\end{tabular}

Source: ORCI for NREL (2001a), Study \#710148, N=941.

Q2.1.4: An energy crisis occurred 25 years ago, in 1973, when the United States imported about one-third of its oil from foreign sources and that oil was shut off. Today, what percentage, from zero to one hundred, of its oil do you think the United States imports from foreign sources?

Table 2.1.4. Public Perception of Imported Oil Share

\begin{tabular}{lcr}
\hline $\begin{array}{l}\text { Percent of Oil Used in U.S. that is } \\
\text { Imported }\end{array}$ & Number & Percent \\
\hline $0 \%$ to $20 \%$ & 52 & 5 \\
$21 \%$ to $40 \%$ & 152 & 15 \\
$41 \%$ to $50 \%$ & 237 & 24 \\
$51 \%$ to $60 \%$ & 120 & 12 \\
$61 \%$ to $70 \%$ & 134 & 13 \\
$71 \%$ to $80 \%$ & 215 & 21 \\
$81 \%$ to $100 \%$ & 77 & 8 \\
Don't Know & 16 & 2 \\
Total & 1,003 & 100 \\
\hline
\end{tabular}

Source: Research/Strategy/Management, Inc., (1998), N=1,003. 


\subsection{PUBLIC ASSESSMENT OF THE ENERGY SITUATION}

Q2.2.1: In fact, the United States imports about half of its oil from foreign sources-more than it did 25 years ago. Based on this fact, how vulnerable do you believe the United States is to an energy crisis that would be caused by foreign nations shutting off their supply of oil to the United States: very, somewhat, not too, not at all?

Table 2.2.1. Public Perception of U.S. Vulnerability to Energy Crisis

\begin{tabular}{lcc}
\hline Categories of Responses & Number & Percent \\
\hline Very vulnerable & 471 & 47 \\
Somewhat vulnerable & 364 & 36 \\
Not too vulnerable & 118 & 12 \\
Not at all vulnerable & 35 & 3.5 \\
Don't know/refused & 15 & 1.5 \\
Total & 1,003 & 100 \\
\hline
\end{tabular}

Source: Research/Strategy/Management, Inc., (1998), N=1,003.

Q2.2.2: How serious would you say the energy situation is in the United States: very serious, fairly serious, or not at all serious?

Table 2.2.2. Public Perception of the Energy Situation in the Year 2001

\begin{tabular}{|l|c|c|c|}
\hline $\begin{array}{l}\text { The Energy Situation in } \\
\text { the U.S. is: }\end{array}$ & $\begin{array}{c}\mathbf{2 0 0 1} \\
\text { March 5-7 } \\
(\boldsymbol{\%})\end{array}$ & $\begin{array}{c}\mathbf{2 0 0 1} \\
\text { May 7-9 } \\
(\boldsymbol{\%})\end{array}$ & $\begin{array}{c}\mathbf{2 0 0 1} \\
\text { June 28 - July 1 } \\
(\boldsymbol{\%})\end{array}$ \\
\hline Very serious & 31 & 58 & 47 \\
Fairly serious & 59 & 36 & 43 \\
Not at all serious & 9 & 4 & 8 \\
No opinion & 1 & 2 & 2 \\
\hline Total & 100 & 100 & 100 \\
\hline
\end{tabular}

Sources: Gallup Poll (2001a, N=1,014; 2001b, N=1,005; 2001c, N=1,060). 
Q2.2.3: Do you think the United States is heading into an energy crisis? Do you think the United States is in an energy crisis now?

Table 2.2.3. Public Perception of the Energy Crisis

\begin{tabular}{lccc}
\hline The United States Is & Yes $(\boldsymbol{\%})$ & No $(\boldsymbol{\%})$ & Total $(\boldsymbol{\%})$ \\
\hline Heading into an energy crisis & 61 & 36 & 97 \\
In an energy crisis & 39 & 60 & 99 \\
\hline
\end{tabular}

Source: ABC News/Washington Post, (2001), N=1,004.

\subsection{PERCEIVED EFFECTS OF GASOLINE PRICES}

Table 2.3.1. Retail Unleaded Regular Gasoline Price in 2000

(Cents Per Gallon, Including Taxes)

\begin{tabular}{cccccccccccc}
\hline Jan & Feb & March & April & May & June & July & Aug & Sept & Oct & Nov & Dec \\
$\mathbf{2 0 0 0}$ & $\mathbf{2 0 0 0}$ & $\mathbf{2 0 0 0}$ & $\mathbf{2 0 0 0}$ & $\mathbf{2 0 0 0}$ & $\mathbf{2 0 0 0}$ & $\mathbf{2 0 0 0}$ & $\mathbf{2 0 0 0}$ & $\mathbf{2 0 0 0}$ & $\mathbf{2 0 0 0}$ & $\mathbf{2 0 0 0}$ & $\mathbf{2 0 0 0}$ \\
\hline 130.1 & 136.9 & 154.1 & 150.6 & 149.8 & 161.7 & 159.3 & 151.0 & 158.2 & 155.9 & 155.5 & 148.9 \\
\hline
\end{tabular}

Source: U.S. DOE/EIA (2001a), Table 9.4.

Table 2.3.2. Retail Unleaded Regular Gasoline Price in 2001

(Cents Per Gallon, Including Taxes)

\begin{tabular}{llllllll}
\hline Jan & Feb & March & April & May & June & July & Aug \\
$\mathbf{2 0 0 1}$ & $\mathbf{2 0 0 1}$ & $\mathbf{2 0 0 1}$ & $\mathbf{2 0 0 1}$ & $\mathbf{2 0 0 1}$ & $\mathbf{2 0 0 1}$ & $\mathbf{2 0 0 1}$ & $\mathbf{2 0 0 1}$ \\
\hline 147.2 & 148.4 & 144.7 & 156.4 & 172.9 & 164.0 & 148.2 & 142.7 \\
\hline
\end{tabular}

Source: U.S. DOE/EIA (2001a), Table 9.4. 
Figure 1. VMT and Gasoline Price Change from Same Period Previo us Year

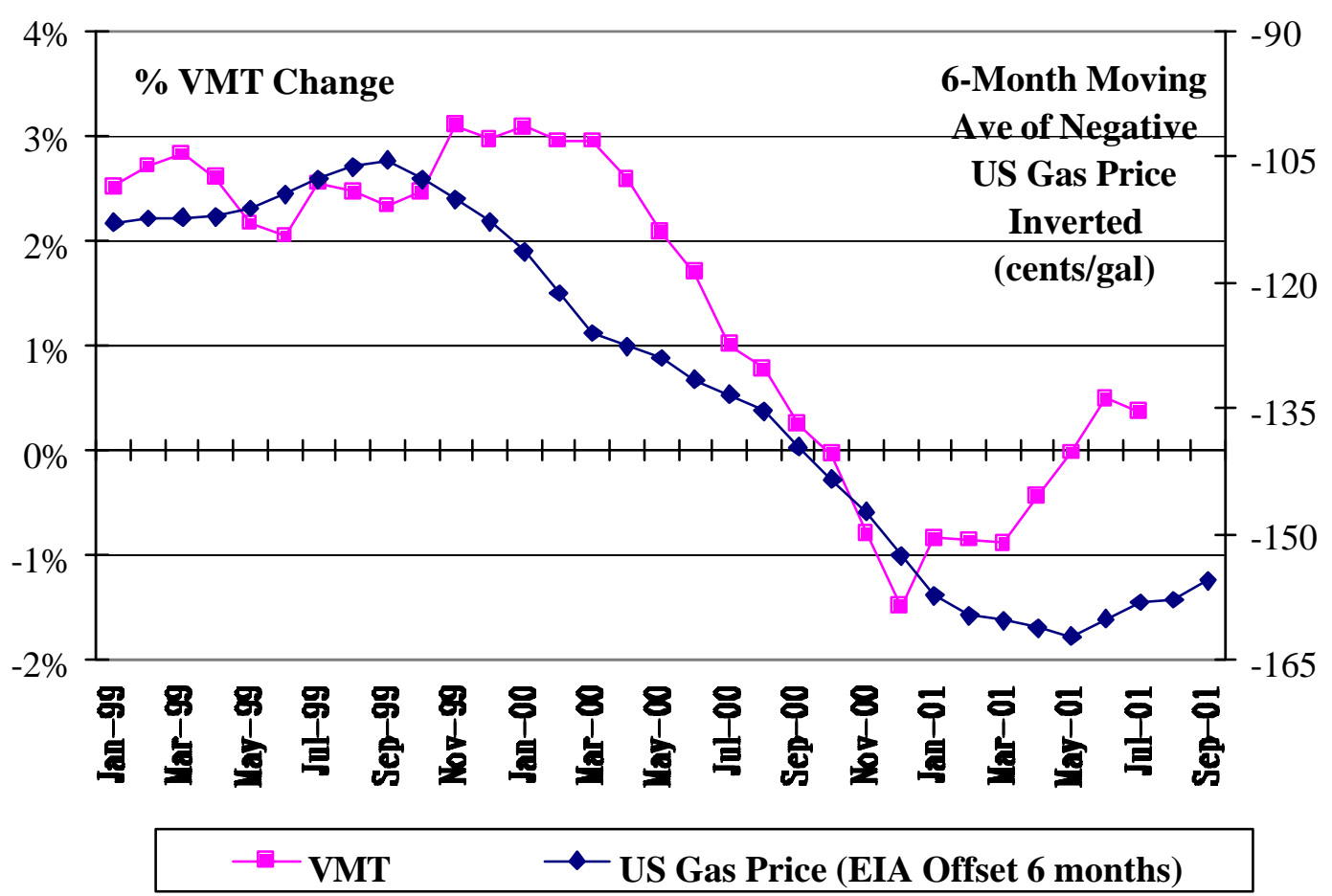

VMT, Vehicles Miles Traveled; Ave, average.

Q2.3.3: Will the price of gas cause you to drive less than you might have otherwise this summer, or not?

Table 2.3.3. Perceived Effects of Gasoline Price Increases on Driving Summer 2001

\begin{tabular}{lccc}
\hline Driving Expectations & $\begin{array}{c}\mathbf{2 0 0 0} \\
\text { May 23-24 } \\
(\mathbf{\%})\end{array}$ & $\begin{array}{c}\mathbf{2 0 0 0} \\
\text { June 22-25 } \\
(\mathbf{\%})\end{array}$ & $\begin{array}{c}\mathbf{2 0 0 1} \\
\text { May 7-9 } \\
\mathbf{( \% )}\end{array}$ \\
\hline $\begin{array}{c}\text { The price of gas will cause me to drive less than } \\
\text { I might have otherwise this summer. }\end{array}$ & 41 & 50 & 58 \\
$\begin{array}{c}\text { The price of gas will not cause me to drive less } \\
\text { than I might have otherwise this summer. }\end{array}$ & 57 & 49 & 41 \\
No opinion & 2 & 1 & 1 \\
\hline Total & 100 & 100 & 100 \\
\hline
\end{tabular}

Sources: Gallup Poll (2000a; 2000b; 2001b), N=1,005. 
Q2.3.4: Which, if any, of the following have you done in the last 6 months, or do you plan on doing in the near future, to reduce gasoline expenditures? (Multiple answers allowed.)

Table 2.3.4. Actions to Reduce Gasoline Expenditures in the Last Six Months (February-August 2000) or Planned for the Near Future

\begin{tabular}{lcc}
\hline Actions To Reduce Gasoline & Number & Percent \\
Expenditures & 718 & 76 \\
\hline Any (net) & 424 & 45 \\
Drive less & 267 & 28 \\
Walk or bike & 239 & 25 \\
Purchase more fuel-efficient vehicle & 198 & 21 \\
Carpool & 162 & 17 \\
Drive a different vehicle than usual & 137 & 15 \\
Begin or increase telecommuting & 123 & 13 \\
Use mass transit more often & 94 & 10 \\
Cancel a vacation trip & 19 & 2 \\
Other & 203 & 22 \\
Nothing & 20 & 2 \\
Don't know & 941 & 100 \\
\hline Total
\end{tabular}

Source: ORCI for NREL (2000a), Study \#709318, N=941.

Q2.3.5: Have recent price increases in gasoline caused any financial hardship for you or your household?

Table 2.3.5. Perceived Effects of Recent Gasoline Price Increases

\begin{tabular}{lccc}
\hline $\begin{array}{l}\text { Effects of Recent Price Increase In } \\
\text { Gasoline }\end{array}$ & $\begin{array}{c}\mathbf{2 0 0 0} \\
\text { May 23-24 } \\
(\mathbf{\%})\end{array}$ & $\begin{array}{c}\mathbf{2 0 0 0} \\
\text { June 22-25 } \\
(\mathbf{\%})\end{array}$ & $\begin{array}{c}\mathbf{2 0 0 1} \\
\text { May 7-9 } \\
(\mathbf{\%})\end{array}$ \\
\hline $\begin{array}{l}\text { Recent price increases in gasoline have } \\
\text { caused financial hardship for my } \\
\text { household or me. }\end{array}$ & 36 & 44 & 47 \\
\hline $\begin{array}{l}\text { Recent price increases in gasoline have } \\
\text { not caused financial hardship for my } \\
\text { household or me. }\end{array}$ & 64 & 56 & 53 \\
\hline Total & 100 & 100 & 100 \\
\hline
\end{tabular}

Sources: Gallup Poll (2000a; 2000b), N=1,014; (2001b), N=1,005. 
Q2.3.6: Do you now drive your vehicle more because of the current low price of gasoline?

Table 2.3.6. Effects of Low Gasoline Prices on Driving - Winter 1999

\begin{tabular}{lcc}
\hline Effects of Low Price of Gasoline On Driving & Number & Percent \\
\hline $\begin{array}{l}\text { Drive my vehicle more now because of the current } \\
\text { low price of gasoline }\end{array}$ & 130 & 13 \\
$\begin{array}{l}\text { Do not drive my vehicle more now because of the } \\
\text { current low price of gasoline }\end{array}$ & 815 & 82 \\
Don't know/don't own vehicle & 54 & 5 \\
\hline Total & 999 & 100 \\
\hline
\end{tabular}

Source: ORCI for NREL (1999a), Study \#70809, N=1,000.

Q2.3.7: On average, how many miles extra are you driving your vehicle per year (record number)?

Table 2.3.7. Average Number of Additional Miles Driven per Year Due to Low Gasoline Prices - Winter 1999

\begin{tabular}{lcc}
\hline Average Number of Additional Miles & Number & Percent \\
\hline $1-500$ & 33 & 25 \\
$501-1,000$ & 14 & 11 \\
$1,001-2,000$ & 21 & 16 \\
$2,001-5,000$ & 26 & 20 \\
More than 5,000 & 16 & 12 \\
Don't know & 21 & 16 \\
\hline Total & 131 & 100 \\
\hline Mean $^{1}$ & 3,535 & \\
\hline Standard deviation & 5,251 & \\
\hline
\end{tabular}

Source: ORCI for NREL (1999), Study \#70809, N=130.

${ }^{1}$ In this report, calculation of means, medians and standard deviations are based on raw numbers. "Don't know" responses are not part of the calculations. 
Q2.3.8: Do you think the current rise in gasoline prices represents a temporary fluctuation in prices or a more permanent change in prices?

Table 2.3.8. Public Assessment of Nature of Current Rise in Gasoline Prices

\begin{tabular}{lccccc}
\hline $\begin{array}{l}\text { Current Rise in Gasoline } \\
\text { Prices Represents }\end{array}$ & $\begin{array}{c}\mathbf{2 0 0 0} \\
\text { Mar 10-12 } \\
(\mathbf{\%})\end{array}$ & $\begin{array}{c}\mathbf{2 0 0 0} \\
\text { Mar 30-Apr 2 } \\
(\mathbf{\%})\end{array}$ & $\begin{array}{c}\mathbf{2 0 0 0} \\
\text { May 23-24 } \\
(\mathbf{\%})\end{array}$ & $\begin{array}{c}\mathbf{2 0 0 0} \\
\text { June 22-25 } \\
(\mathbf{\%})\end{array}$ & $\begin{array}{c}\mathbf{2 0 0 1} \\
\text { May 7-9 } \\
(\mathbf{\%})\end{array}$ \\
\hline $\begin{array}{l}\text { Temporary fluctuation in } \\
\text { prices }\end{array}$ & 63 & 60 & 45 & 57 & 40 \\
$\begin{array}{l}\text { More permanent change in } \\
\text { prices }\end{array}$ & 34 & 37 & 50 & 39 & 56 \\
No opinion & 3 & 3 & 5 & 4 & 4 \\
\hline Total & 100 & 100 & 100 & 100 & 100 \\
\hline
\end{tabular}

Sources: Gallup Poll (2000d), N=500; (2000c), N=500; (2000b), N=500; (2000a), N=500; (2001b), $\mathrm{N}=500)$.

Q2.3.9: Looking ahead to one month from now, do you think gas prices at that time will be: higher than they are today, about the same, or lower than they are today?

Looking ahead to six months from now, do you think gas prices at that time will be: higher than they are today, about the same, or lower than they are today?

Table 2.3.9. Public Perception of Gas Prices One to Six Months from Today

\begin{tabular}{|c|c|c|c|c|c|c|c|c|}
\hline \multirow{2}{*}{$\begin{array}{l}\text { Looking Ahead } \\
\text { From Now, Gas } \\
\text { Prices Will Be }\end{array}$} & \multicolumn{4}{|c|}{$\begin{array}{c}\text { One Month From Now, Do } \\
\text { You Think Gas Prices Will Be } \\
(\%)\end{array}$} & \multicolumn{4}{|c|}{$\begin{array}{c}\text { Six Months From Now, Do } \\
\text { You Think Gas Prices Will Be } \\
(\%)\end{array}$} \\
\hline & $\begin{array}{c}2000 \\
\text { March } \\
10-12\end{array}$ & $\begin{array}{l}2000 \\
\text { May } \\
23-24\end{array}$ & $\begin{array}{l}2000 \\
\text { June } \\
22-25\end{array}$ & $\begin{array}{c}2001 \\
\text { May } \\
7-9\end{array}$ & $\begin{array}{c}2000 \\
\text { March } \\
10-12\end{array}$ & $\begin{array}{l}2000 \\
\text { May } \\
23-24\end{array}$ & $\begin{array}{l}2000 \\
\text { June } \\
22-25\end{array}$ & $\begin{array}{c}2001 \\
\text { May } \\
7-9\end{array}$ \\
\hline $\begin{array}{l}\text { Higher than they } \\
\text { are today }\end{array}$ & 74 & 51 & 38 & 83 & 37 & 24 & 20 & 38 \\
\hline About the same & 16 & 33 & 39 & 13 & 26 & 25 & 28 & 37 \\
\hline $\begin{array}{c}\text { Lower than they } \\
\text { are today }\end{array}$ & 9 & 14 & 22 & 3 & 34 & 49 & 50 & 24 \\
\hline No opinion & 1 & 2 & 1 & 1 & 3 & 2 & 2 & 1 \\
\hline Total & 100 & 100 & 100 & 100 & 100 & 100 & 100 & 100 \\
\hline
\end{tabular}

Sources: Gallup Poll (2000d), N=505; (2000b), N=505; (2000a), N=505; (2001b), N=505. 
Q2.3.10: How concerned are you about the price you will pay for gasoline over the next year? Would you say: very concerned, somewhat concerned, or not at all concerned?

Table 2.3.10. Public Concern About the Price of Gasoline in 2002

\begin{tabular}{lcc}
\hline Degree of Public Concern & Number & Percent \\
\hline Very concerned & 462 & 49 \\
Somewhat concerned & 334 & 36 \\
Not at all concerned & 142 & 15 \\
Don't know & 3 & small base \\
\hline Total & 941 & 100 \\
\hline
\end{tabular}

Source: ORCI for NREL (2001a). Study \#710148, N=941.

\subsection{PUBLIC BELIEFS ABOUT ACTIONS TO ADDRESS THE ENERGY PROBLEMS}

Q2.4.1: Which of the following approaches to solving the nation's energy problems do you think the United States should follow right now: emphasize production of more oil, gas and coal supplies, or emphasize more conservation by consumers of existing energy supplies?

Table 2.4.1. Public Preference for Solving the Nation's Energy Problems

\begin{tabular}{lcc}
\hline $\begin{array}{l}\text { Approaches to Solving the Nation's Energy Problems the US } \\
\text { Should Follow Now }\end{array}$ & $\begin{array}{c}\mathbf{2 0 0 1} \\
\text { March 5-7 } \\
(\mathbf{\%})\end{array}$ & $\begin{array}{c}\mathbf{2 0 0 1} \\
\text { May 7-9 } \\
(\mathbf{\%})\end{array}$ \\
\hline Emphasize production of more oil, gas, and coal supplies & 33 & 35 \\
Emphasize more conservation by consumers of existing energy & 56 & 47 \\
$\quad$ supplies & & \\
Both/equally & 8 & 14 \\
Neither/other & 1 & 2 \\
No opinion & 2 & 2 \\
\hline Total & 100 & 100 \\
\hline
\end{tabular}

Sources: Gallup Poll (2001c), N=505; (2001b), N=505. 
Q2.4.2: If it became more important for the United States to reduce dependence on imported oil, what actions do you think should be taken by individuals, government, and/or business? (open-ended)

Table 2.4.2. Public Perception of Actions that Should Be Taken by Individuals, Government, and/or Business to Reduce Dependence on Imported Oil

\begin{tabular}{|c|c|c|c|}
\hline $\begin{array}{l}\text { Actions that Should } \\
\text { and/or Business' To }\end{array}$ & $\begin{array}{l}\text { Be Taken By Individuals, Government, } \\
\text { Reduce Dependence on Imported Oil }\end{array}$ & Number & Percent \\
\hline $\begin{array}{l}\text { Conservation/reduce } \\
\text { consumption (net) }\end{array}$ & & 229 & 24 \\
\hline & $\begin{array}{l}\text { Conservation/reduce consumption } \\
\quad \text { (unspecified) }\end{array}$ & 129 & 14 \\
\hline & Research/use more fuel-efficient cars & 34 & 4 \\
\hline & Carpool & 25 & 3 \\
\hline & Provide/use public transportation & 24 & 3 \\
\hline & Drive less/walk/bike more/ration gas & 31 & 3 \\
\hline & $\begin{array}{l}\text { All other conservation/reduce } \\
\text { consumption mentions }\end{array}$ & 22 & 2 \\
\hline Other sources of oil & Drilling, use U.S. oil reserves & 195 & 21 \\
\hline $\begin{array}{l}\text { Alternative energy } \\
\text { sources (net) }\end{array}$ & & 167 & 18 \\
\hline & $\begin{array}{l}\text { Research/use alternative energy sources } \\
\quad \text { (unspecified) }\end{array}$ & 93 & 10 \\
\hline & $\begin{array}{l}\text { Research/ Use our own natural } \\
\text { resources }\end{array}$ & 16 & 2 \\
\hline & Research/use electric cars & 16 & 2 \\
\hline & $\begin{array}{l}\text { Research/use cars that don't use gas but } \\
\text { use solar energy, nuclear power, wind } \\
\text { power, other alternative energy sources }\end{array}$ & 55 & 5 \\
\hline Change prices & & 39 & 4 \\
\hline $\begin{array}{l}\text { Government } \\
\text { involvement }\end{array}$ & Government/Government's responsibility & 38 & 4 \\
\hline $\begin{array}{l}\text { Environmental } \\
\text { concerns }\end{array}$ & & 23 & 2 \\
\hline Other & & 107 & 12 \\
\hline Nothing & & 48 & 5 \\
\hline Don't know & & 206 & 22 \\
\hline Total & & 941 & 112 \\
\hline
\end{tabular}

Source: ORCI for NREL (2001a), Study \#710148, N=941. 
Q2.4.3: Which, if any, of the following have you done in the last 6 months, or do you plan on doing in the near future, to reduce gasoline expenditures?

Table 2.4.3. Actions Taken to Reduce Oil Dependence in the Last Six Months (February-August 2000) or Planned to Be Taken in the Near Future

\begin{tabular}{lrr}
\hline $\begin{array}{l}\text { Actions To Reduce Gasoline } \\
\text { Expenditures }\end{array}$ & Number & Percent \\
\hline Any (net) & 718 & 76 \\
Drive less & 424 & 45 \\
Walk or bike & 267 & 28 \\
Purchase more fuelefficient vehicle & 239 & 25 \\
Carpool & 198 & 21 \\
Drive a different vehicle than usual & 162 & 17 \\
Begin or increase telecommuting & 137 & 15 \\
Use mass transit more often & 123 & 13 \\
Cancel a vacation trip & 94 & 10 \\
Other & 19 & 2 \\
Nothing & 203 & 22 \\
Don't know & 20 & 2 \\
\hline Total & 941 & 100 \\
\hline
\end{tabular}

Source: ORCI for NREL (2000a), Study \#709318, N=941. 
Q2.4.4: In order to make our country less dependent on oil from insecure regions in the world, citizens like you could help by reducing the amount of fuel your vehicle consumes by one gallon per week. Which one of the following would you most likely do to save one gallon of fuel per week?

Table 2.4.4. Public Preference for Saving One Gallon of Fuel per Week

\begin{tabular}{|c|c|c|c|}
\hline \multicolumn{2}{|c|}{ Actions to Save One Gallon of Fuel Per Week } & \multirow{2}{*}{$\begin{array}{r}\text { Number } \\
569\end{array}$} & \multirow{2}{*}{$\begin{array}{r}\text { Percent } \\
57\end{array}$} \\
\hline Any (net) & & & \\
\hline & $\begin{array}{l}\text { Use mass transit or carpool to get to } \\
\text { work }\end{array}$ & 283 & 28 \\
\hline & $\begin{array}{l}\text { Purchase a vehicle that gets ten percent } \\
\text { better fuel economy than the } \\
\text { one you currently drive }\end{array}$ & 180 & 18 \\
\hline & $\begin{array}{l}\text { Work at home one or two days per } \\
\text { week by telecommuting }\end{array}$ & 106 & 11 \\
\hline $\begin{array}{l}\text { Would do something else to } \\
\text { reduce fuel consumption }\end{array}$ & & 283 & 28 \\
\hline $\begin{array}{l}\text { Not interested in saving one } \\
\text { gallon of fuel per week }\end{array}$ & & 33 & 3 \\
\hline Don't drive/don't have a car & & 60 & 6 \\
\hline Don't know & & 55 & 5 \\
\hline Total & & 1,000 & 99 \\
\hline
\end{tabular}

Source: ORCI for NREL (2001b), Study \#710449, N=1,000. 
Q2.4.5: What would you do to save fuel? (open-ended)

Table 2.4.5. Possible Actions Taken by Individuals to Reduce Fuel Consumption

\begin{tabular}{llrr}
\hline Possible Actions by to Reduce Fuel Consumption & Number & Percent \\
\hline Drive less (net) & & 126 & 45 \\
& Drive less & 38 & 13 \\
& Consolidate trips & 24 & 8 \\
& Run fewer errands/trips & 19 & 7 \\
& Drive only when necessary/reduce needless & 10 & 3 \\
& travel & & \\
& Travel less & 10 & 3 \\
& Less weekend/pleasure driving & 14 & 4 \\
& All other drive less mentions & 12 & 4 \\
\hline Walk (net) & & 46 & 16 \\
& Walk (unspecified) & 33 & 12 \\
& Walk to work & 8 & 3 \\
& All other walk mentions & 5 & 2 \\
\hline Stay home (net) & & 24 & 8 \\
& Stay home more often & 10 & 3 \\
Ride bike & All other stay home mentions & 14 & 5 \\
\hline Keep car tuned & & 11 & 4 \\
up/maintained & & 8 & 3 \\
properly & & & 12 \\
\hline Other & & 34 & 12 \\
\hline Total & & 34 & 12 \\
\hline Source: & & 283 & 100 \\
\hline
\end{tabular}

Source: ORCI for NREL (2001b), Study \#710449, N=283. 
Q2.4.6: Here are some things that can be done to deal with the energy situation. For each one, please say whether you generally favor or oppose it. How about...?

Table 2.4.6. Public Perception of Ways to Deal with the Energy Situation (Gallup Poll)

\begin{tabular}{|c|c|c|c|c|}
\hline Ways to Deal with the Energy Situation & $\begin{array}{c}\text { Favor } \\
(\%)\end{array}$ & $\begin{array}{l}\text { Oppose } \\
(\%)\end{array}$ & $\begin{array}{l}\text { No Opinion } \\
(\%)\end{array}$ & $\begin{array}{c}\text { Total } \\
(\%)\end{array}$ \\
\hline $\begin{array}{l}\text { Investments in new sources of energy such as } \\
\text { solar, wind and fuel cells }\end{array}$ & 91 & 6 & 3 & 100 \\
\hline $\begin{array}{l}\text { Mandating more energy-efficient appliances } \\
\text { such as air conditioning, clothes dryers, } \\
\text { water heaters }\end{array}$ & 87 & 12 & 1 & 100 \\
\hline Mandating more energy-efficient new buildings & 86 & 12 & 2 & 100 \\
\hline Mandating more energy-efficient cars & 85 & 14 & 1 & 100 \\
\hline Investing in new power generating plants & 83 & 13 & 4 & 100 \\
\hline $\begin{array}{l}\text { Federal government partnership with auto } \\
\text { industry working toward energy-efficient } \\
\text { cars }\end{array}$ & 76 & 22 & 2 & 100 \\
\hline Investing in more electrical transmission lines & 69 & 23 & 8 & 100 \\
\hline Investing in more gas pipelines & 64 & 29 & 7 & 100 \\
\hline Drilling for natural gas on federal lands & 63 & 33 & 4 & 100 \\
\hline $\begin{array}{l}\text { Increasing the use of nuclear power as a major } \\
\text { source of power }\end{array}$ & 48 & 44 & 8 & 100 \\
\hline $\begin{array}{l}\text { Opening up the Alaskan Arctic Wildlife Refuge } \\
\text { for oil exploration }\end{array}$ & 38 & 57 & 5 & 100 \\
\hline
\end{tabular}

Source: Gallup Poll (2001b), N=505. 
Q2.4.7: Here are several proposals that have been made to help solve America's energy problems. Do you favor or oppose each one?

Table 2.4.7. Public Perception of Ways to Deal with the Energy Situation (NBC/WSJ)

\begin{tabular}{|c|c|c|c|c|}
\hline Ways to Deal with the Energy Situation & $\begin{array}{c}\text { Favor } \\
(\%)\end{array}$ & $\begin{array}{l}\text { Oppose } \\
(\%)\end{array}$ & $\begin{array}{c}\text { No } \\
\text { Opinion } \\
(\%)\end{array}$ & $\begin{array}{c}\text { Total } \\
(\%)\end{array}$ \\
\hline $\begin{array}{l}\text { Require automakers to produce more fuel- } \\
\text { efficient cars }\end{array}$ & 87 & 10 & 3 & 100 \\
\hline $\begin{array}{l}\text { Financial incentives for business, consumers to } \\
\text { conserve energy }\end{array}$ & 85 & 12 & 3 & 100 \\
\hline $\begin{array}{l}\text { Make permitting and building new power plants } \\
\text { easier }\end{array}$ & 69 & 24 & 7 & 100 \\
\hline Place federal price controls on gasoline & 56 & 38 & 6 & 100 \\
\hline $\begin{array}{l}\text { Place federal price controls on electricity and } \\
\text { natural gas }\end{array}$ & 54 & 41 & 5 & 100 \\
\hline $\begin{array}{l}\text { Place mandatory conservation regulations on } \\
\text { businesses and consumers }\end{array}$ & 53 & 42 & 5 & 100 \\
\hline $\begin{array}{l}\text { Allow drilling for oil, gas in Alaskan Arctic } \\
\text { National Wildlife Refuge }\end{array}$ & 43 & 50 & 7 & 100 \\
\hline Relax clean air, environmental standards & 30 & 65 & 5 & 100 \\
\hline
\end{tabular}

Source: NBC/Wall Street Journal, June 23-25, 2001, N=806. 
Q2.4.8: For U.S. energy needs, do you support or oppose Federal Government action to...?

Table 2.4.8. Public Support of Federal Government Actions for U.S. Energy Needs

\begin{tabular}{lcccc}
\hline $\begin{array}{l}\text { Federal Government Actions For U.S. } \\
\text { Energy Needs }\end{array}$ & $\begin{array}{c}\text { Support } \\
(\mathbf{\%})\end{array}$ & $\begin{array}{c}\text { Oppose } \\
(\mathbf{\%})\end{array}$ & $\begin{array}{c}\text { No Opinion } \\
(\mathbf{\%})\end{array}$ & $\begin{array}{c}\text { Total } \\
(\mathbf{\%})\end{array}$ \\
\hline $\begin{array}{l}\text { Develop more solar and wind power } \\
\begin{array}{l}\text { Encourage more energy conservation by } \\
\text { businesses and industries }\end{array}\end{array}$ & 90 & 8 & 2 & 100 \\
$\begin{array}{l}\text { Encourage more energy conservation by } \\
\quad \text { consumers like yourself }\end{array}$ & 90 & 8 & 2 & 100 \\
$\begin{array}{l}\text { Require car manufacturers to improve } \\
\quad \text { fuel efficiency of vehicles sold in the }\end{array}$ & $\mathbf{8 9}$ & $\mathbf{1 0}$ & $\mathbf{1}$ & $\mathbf{1 0 0}$ \\
$\quad$ United States & & & & 100 \\
$\begin{array}{l}\text { Increase oil and gas drilling } \\
\text { Build more power plants that burn oil, coal } \\
\text { or natural gas }\end{array}$ & 62 & 31 & 7 & 100 \\
$\begin{array}{l}\text { Increase coal mining } \\
\text { Build more nuclear power plants }\end{array}$ & 54 & 39 & 7 & 100 \\
\hline
\end{tabular}

Source: ABC News/Washington Post, May 31-June 3, 2001, N=1,004.

Q2.4.9: Which one should be the Federal Government highest priority?

Table 2.4.9. Public Perception of the Federal Government's Highest Priority

Federal Government Highest Priority

Percent

\begin{tabular}{ll}
\hline Develop more solar and wind power & 23
\end{tabular}

Require car manufacturers to improve fuel efficiency of vehicles sold in the United States

Encourage more energy conservation by businesses and industries 17

Increase oil and gas drilling $\quad 11$

Build more power plants that burn oil, coal or natural gas $\quad 10$

Encourage more energy conservation by consumers like yourself $\quad 8$

$\begin{array}{ll}\text { Build more nuclear power plants } & 8\end{array}$

$\begin{array}{lr}\text { Increase coal mining } & 1\end{array}$

\begin{tabular}{lc}
\hline Total & 97 \\
\hline
\end{tabular}

Source: ABC News/Washington Post, May 31-June 3, 2001, N=1,004. 
Q2.4.10: If it became important for the United States to reduce dependence on imported oil, which of the following policies would you most support?

If it became important for the United States to reduce dependence on imported oil, which of the following policies would you least support?

Table 2.4.10. Policies the Public Would Most/Least Support to Reduce

Dependence on Imported Oil

\begin{tabular}{lcccc}
\hline $\begin{array}{l}\text { Policies To Reduce Dependence On } \\
\text { Imported Oil }\end{array}$ & \multicolumn{2}{c}{ Most Support } & \multicolumn{2}{c}{ Least Support } \\
\cline { 2 - 5 } & Number & Percent & Number & Percent \\
\hline $\begin{array}{l}\text { Regulation to make personal vehicles } \\
\text { more efficient }\end{array}$ & $\mathbf{4 5 5}$ & $\mathbf{4 8}$ & $\mathbf{1 4 5}$ & $\mathbf{1 5}$ \\
$\begin{array}{l}\text { Tax refunds for higher efficiency vehicles } \\
\text { Higher taxes on less efficient vehicles }\end{array}$ & 223 & 24 & 134 & 14 \\
Higher taxes on gasoline and diesel & 101 & 11 & 174 & 19 \\
Don't know/none of these & 58 & 6 & 418 & 44 \\
\hline Total & 104 & 11 & 69 & 7 \\
\hline
\end{tabular}

Source: ORCI for NREL (2001a), Study \#710148, N=941. 


\section{GLOBAL WARMING}

A number of surveys ${ }^{1}$ researched the U.S. population's awareness of global warming. In general, the public is well aware of global warming. In 1998, a majority of the population had heard about global warming (Table 3.1.1). However, they were not so aware of the Kyoto ${ }^{2}$ conference on global warming. Almost three quarters of Americans said they had heard "not very much" or "nothing at all" about the Kyoto Conference (Table 3.1.2).

A majority of the public believes that global warming is a real problem. Two-thirds of the adult population perceived the global warming threat as serious in 1997 (Table 3.1.3). U.S. adults assess global warming as the third (of the five given choices) most important transportation problem to the United States in the year of 2020, following traffic congestion and availability and/or price of gasoline (Table 3.1.4).

An overwhelming majority of Americans support taking actions that incur costs in order to combat global warming. However, in 1998 the public believed that the problem of global warming could be dealt with gradually by taking steps which were low in cost (Table 3.2.1). When asked about a policy to reduce greenhouse gas emissions from vehicles, an overwhelming majority of U.S. adults would prefer a 3 percent tax for new vehicles rather than a 25-cent per gallon tax on gasoline (Table 3.2.2).

Americans believe that the United States should join other countries in setting standards to improve the global environment (Table 3.2.3). In 1998, the public expected that less-developed countries should be required to limit their emissions (Table 3.2.4). Americans favored limits in increases in emissions in less-developed countries rather than cuts in these countries' emissions (Table 3.2.5).

\footnotetext{
${ }^{1}$ The surveys were conducted by the Pew Research Center for the People \& the Press (1997), the Sustainable Energy Budget Coalition (1996), the World Wildlife Fund National Survey (1997), the New York Times (November 23-24, 1997), and the Program on International Policy Attitudes (PIPA, 1998a; 1998b).

${ }^{2}$ The Kyoto conference on global warming took place in Japan in December 1997. At the conference the United States and Europe agreed to cut their greenhouse gas emissions by 7-8 percent below the 1990 level by about the year 2010 .
} 


\subsection{PUBLIC AWARENESS AND PERCEIVED SERIOUSNESS OF THE GLOBAL WARMING ISSUE}

Q3.1.1: WWF: How much, if anything, have you heard or read about the issue of global warming? Would you say you have heard a great deal, some, not too much or nothing at all about global warming?

PIPA: As you may know in the next few weeks there will be a world conference in Buenos Aires on the problem of global warming caused by greenhouse gas emissions, which are released by the burning coal, gasoline, and other fossil fuels. How much have you heard about global warming: a great deal, some, not very much or nothing at all?

Table 3.1.1. Level of Public Awareness of Global Warming

\begin{tabular}{lcc}
\hline Degree of Awareness & WWF (\%) & PIPA (\%) \\
\hline A lot & 25 & 32 \\
Some & 46 & 39 \\
Not much/nothing & 29 & 29 \\
\hline Total & 100 & 100 \\
\hline Sources: World Wildlife Fund National Survey (1997), and PIPA (1998b), N=800.
\end{tabular}

Q3.1.2: As you may know recently there was a world conference in Kyoto on the problem of global warming. How much have you heard about this conference?

Table 3.1.2. Level of Public Awareness of the Kyoto Conference

\begin{tabular}{lc}
\hline Degree of Awareness & Percent \\
\hline A great deal & 5 \\
Some & 20 \\
Not very much & 39 \\
Nothing & 35 \\
\hline Total & 99 \\
\hline
\end{tabular}

Source: PIPA (1998a), N=648, margin of error $+/-3.5-4 \%$. 
Q3.1.3: $\quad$ SEBC: In your mind, how serious a threat do you think global climate change, also known as global warming, caused by emissions from the combustion of oil, gasoline, and coal is?

WWF: Generally speaking, how serious of a threat do you think global warming is today: very serious, somewhat serious, not too serious, or not serious at all or don't you have an opinion on this?

Table 3.1.3. Perceived Seriousness of Global Warming Threat

\begin{tabular}{lcc}
\hline Degree of Seriousness & SEBC (\%) & WWF (\%) \\
\hline Very serious & 36 & 24 \\
Somewhat serious & 35 & 42 \\
Not too serious & 16 & 12 \\
Not serious at all & 9 & 7 \\
Don't know & 4 & 14 \\
\hline Total & 100 & 99 \\
\hline
\end{tabular}

Sources: Sustainable Energy Budget Coalition (1996), and World Wildlife Fund National Survey (1997).

Q3.1.4: Thinking about the future, which of the following transportation problems will be most important to the United States in the year 2020?

Table 3.1.4. Public Ranking of the Most Important Transportation Problem for the United States in the Year 2020

\begin{tabular}{lcc}
\hline Most Important Transportation Problem & Number & Percent \\
\hline Traffic congestion & 339 & 34 \\
Availability and/or price of gasoline & 282 & 28 \\
Global warming or climate change caused by & 140 & 14 \\
$\quad$ vehicles & 122 & 12 \\
Local air pollution from vehicles & 74 & 7 \\
Deaths and serious injuries in vehicle accidents & 42 & 4 \\
Don't know & 999 & 99 \\
\hline Total
\end{tabular}

Source: ORCI for NREL, (2000b), Study \#709489, N=1,000. 


\subsection{PERCEIVED ACTIONS TO ADDRESS GLOBAL WARMING}

Q3.2.1: There is a controversy over what the countries of the world, including the United States, should do about the problem of global warming. I am going to read you three statements. Please, tell me which statement comes closest to your point of view.

Table 3.2.1. Public Support for Actions to Address Global Warming

\section{Action to Address Global Warming}

February-April 1998

$(\%)$
October 1998

$(\%)$

Until we are sure that global warming is really a problem, we should not take any steps that would have economic costs.

The problem of global warming should be addressed, but its effects will be gradual, so we can deal with the problem gradually by taking steps that are low in cost.

Global warming is a serious and pressing problem.

We should begin taking steps now even if this involves significant costs.

Don't know/refused

2

100

Source: PIPA (1998a), N=600 and PIPA (1998b), N=800.

Q3.2.2: If the nation determines that it is important to reduce greenhouse gas emissions from vehicles, which of the following policies would you prefer?

Table 3.2.2. Public Preference for Policy to Reduce Greenhouse Gas Emissions from Vehicles

\begin{tabular}{lrr}
\hline $\begin{array}{l}\text { Policy to Reduce Greenhouse Gas Emissions } \\
\text { from Vehicles }\end{array}$ & Number & Percent \\
\hline 25-cent per gallon tax on gasoline & 171 & 17 \\
3\% tax for new vehicles & 700 & 70 \\
Don't know/none of these & 129 & 13 \\
\hline Total & 1,000 & 100 \\
\hline
\end{tabular}

Source: ORCI for NREL (1998a), Study \#707089, N=1,000. 
Q3.2.3: Do you think the United States should join other countries in setting standards to improve the global environment, or should the United States set its own environmental standards independently?

Table 3.2.3. Preferred Strategies for Combating Global Warming

\begin{tabular}{lc}
\hline U.S. Strategies & Percent \\
\hline Join other countries & 55 \\
Set standards independently & 41 \\
Don't know/refused & 4 \\
\hline Total & 100 \\
\hline
\end{tabular}

Source: Pew Research Center for the People \& the Press (1997).

Q3.2.4: Another controversy is whether the less economically developed countries should also be expected to cut their emissions of the greenhouse gasses that cause global warming. Please tell me which of the following positions comes closest to yours?

Table 3.2.4. Perceived Contribution by Less-Developed Countries Toward Production of Greenhouse Gas Emissions

\section{Positions on Less-Developed Countries}

Percent

On a per person basis, less-developed countries produce far less greenhouse 38 gases than developed countries. Therefore, the less-developed countries should not be required to limit their emissions until they develop their economies more.

The less-developed countries produce a substantial and growing amount of greenhouse gasses. Therefore they should be required to limit their emissions.

Don't know

Source: PIPA (1998a), N=600. 
Q3.2.5: Another controversy is whether the less developed countries should also be expected to limit or reduce their greenhouse gas emissions. Please tell me which of the following positions comes closest to yours.

Table 3.2.5. Public Perception of Actions Which Should Be Required of Less-Developed Countries to Reduce Greenhouse Gas Emissions

Actions which Should Be Required of Less-Developed Countries Percent

The more-developed countries produce far more greenhouse gas emissions 19 and have not begun to make meaningful reductions. So the lessdeveloped countries should not be required to limit their emissions until the more-developed countries reduce theirs.

The less-developed countries produce a substantial and growing amount of greenhouse gas emissions. Therefore they should be required to cut their emissions.

Because the less-developed countries are poorer and produce far lower emissions, they should not be required to cut back. But they should be required to minimize the increase of their emissions through greater energy efficie ncy.

None of these.

Source: PIPA (1998b), N=800. 


\section{ALTERNATIVE FUELS}

A number of surveys researched the U.S. adult population knowledge and opinions about alternative types of fuel such as electricity, ethanol, and hydrogen. On average, Americans believed in 1998 that gasoline and diesel will become too expensive to use in cars and trucks by the year 2010. In 1998, U.S. adults thought that electricity followed by solar energy would most likely replace gasoline and diesel in the future (Table 4.1.2). Similar to this finding, in 2000, Americans chose electricity over ethanol and hydrogen as the best fuel to use in personal vehicles when gasoline is no longer available (Table 4.1.3). They chose electricity because of environmental concerns (such as electricity being cleaner and less polluting), and its availability (Table 4.1.4).

Those Americans who preferred ethanol to electricity and hydrogen as the best fuel to use when gasoline is no longer available referred to its availability as one of the primary reasons (Table 4.1.5). Those who selected hydrogen as the best fuel to use in personal vehicles in the future also explained their choice by hydrogen's availability, along with environmental concerns (Table 4.1.6).

The same survey addressed the issue of the worst fuel to use in personal vehicles when gasoline is no longer available. Almost three in ten Americans chose ethanol over electricity and hydrogen as the worst fuel for use in the future because of environmental concerns (Tables 4.1.7, 4.1.8). People who rated hydrogen as the worst fuel to use when gasoline is no longer available did so mainly because of safety concerns, such as hydrogen being explosive, flammable/combustible and dangerous/not safe (Table 4.1.9). Those who selected electricity as the worst fuel to use in the future cited electricity being expensive, and environmental concerns (Table 4.1.10). In addition, the U.S. public complained that electric vehicles could not hold a charge for long and, therefore, could not travel long distances. 
Q4.1.1: In what year in the future do you think gasoline and diesel will become too expensive in cars and trucks?

Table 4.1.1. Public Perception of When Gasoline and Diesel Will Become Too Expensive

\begin{tabular}{lrr}
\hline Year & Number & Percent \\
\hline 1999 & 59 & 6 \\
2000 & 87 & 9 \\
$2001-2005$ & 142 & 14 \\
$2006-2010$ & 109 & 11 \\
$2011-2015$ & 27 & 3 \\
$2016-2020$ & 71 & 7 \\
$2021-2025$ & 39 & 4 \\
$2026-2050$ & 70 & 7 \\
2051 or later & 52 & 5 \\
Don't know & 343 & 34 \\
\hline Total & 1,000 & 100 \\
\hline
\end{tabular}

Source: ORCI for NREL (1998b), Study \#707349*, N=1,000.

* Survey responses may have been biased because the question was asked within a week of the U.S. missile attack on terrorists in Sudan and Afghanistan.

Q4.1.2: What fuel will most likely replace gasoline and diesel when they become too expensive to use in cars and trucks? Any others? (open-ended)

Table 4.1.2. Public Perception of Which Fuel Will Replace Gasoline and Diesel

\begin{tabular}{lrc}
\hline Fuel & Number & Percent \\
\hline Electricity/battery & 332 & 33 \\
Solar & 123 & 12 \\
Alcohol/ethanol/methanol & 102 & 11 \\
Natural gas/CNG/LNG & 61 & 6 \\
Hydrogen & 26 & 3 \\
Propane (LPG) & 23 & 2 \\
Water, nuclear & 25 & 3 \\
Other & 54 & 4 \\
Don't know/none & 253 & 25 \\
\hline Total & 1,000 & 99 \\
\hline
\end{tabular}

Source: ORCI for NREL (1998b), Study \#707349, $\mathrm{N}=1,000$. CNG, Compressed Natural Gas; LNG, Liquefied Natural Gas; LPG, Liquid Propane Gas. 
Public Perceptions of Best Fuel for Use in Personal Vehicles When Gasoline Is No Longer Available

Q4.1.3: Consider a future date when gasoline is no longer available. Which of the following do you think would be the best fuel for use in personal vehicles: electricity, ethanol, or hydrogen?

Table 4.1.3. Public Perception of Best Fuel for Use in Personal Vehicles

When Gasoline Is No Longer Available

\begin{tabular}{lcc}
\hline $\begin{array}{l}\text { Best Fuel for Use in } \\
\text { Personal Vehicles }\end{array}$ & Number & Percent \\
\hline Electricity & 522 & 52 \\
Ethanol & 206 & 21 \\
Hydrogen & 151 & 15 \\
Don't know & 121 & 12 \\
\hline Total & 1,000 & 100 \\
\hline
\end{tabular}

Source: ORCI for NREL (2000b), Study $\# 709489^{*}, \mathrm{~N}=1,000$.

* The ORCI study \# 709489 was conducted before the electricity problems in California 
Q4.1.4: Why did you say electricity would be the best fuel for use in personal vehicles when gasoline is no longer available? (open-ended)

Table 4.1.4. Reasons Electricity Would Be the Best Fuel for Use in Personal Vehicles When Gasoline Is No Longer Available

\begin{tabular}{|c|c|c|c|c|c|c|c|c|c|c|c|}
\hline & \multirow{3}{*}{ Reasons } & \multicolumn{2}{|c|}{ Total } & \multicolumn{8}{|c|}{ Region } \\
\hline & & \multirow{2}{*}{$\mathbf{n}$} & \multirow{2}{*}{$\%$} & \multicolumn{2}{|c|}{$\mathrm{NE}^{1}$} & \multicolumn{2}{|c|}{$\mathbf{N} \mathbf{C}^{1}$} & \multicolumn{2}{|c|}{$\mathbf{S}^{1}$} & \multicolumn{2}{|c|}{$\mathbf{W}^{1}$} \\
\hline & & & & $\mathbf{n}$ & $\%$ & $\mathbf{n}$ & $\%$ & $\mathbf{n}$ & $\%$ & $\mathbf{n}$ & $\%$ \\
\hline \multirow{5}{*}{$\begin{array}{l}\text { Environmental concerns } \\
\quad \text { (net) }\end{array}$} & & 153 & 29 & 36 & 32 & 29 & 28 & 48 & 25 & 40 & 35 \\
\hline & Cleaner & 73 & 14 & 19 & 17 & 16 & 15 & 20 & 10 & 18 & 16 \\
\hline & Does not pollute/less pollution & 50 & 10 & 9 & 8 & 10 & 9 & 20 & 10 & 11 & 10 \\
\hline & Less air pollution/cleaner air & 24 & 5 & 8 & 7 & 1 & 1 & 7 & 4 & 8 & 7 \\
\hline & $\begin{array}{l}\text { All other environmental concerns } \\
\text { mentions }\end{array}$ & 24 & 5 & 4 & 3 & 6 & 6 & 6 & 4 & 8 & 8 \\
\hline \multirow[t]{5}{*}{ Availability (net) } & & 117 & 22 & 28 & 25 & 25 & 24 & 40 & 21 & 23 & 20 \\
\hline & $\begin{array}{l}\text { Common/readily available/ } \\
\text { abundant }\end{array}$ & 64 & 12 & 13 & 11 & 15 & 14 & 23 & 12 & 14 & 12 \\
\hline & Renewable/inexhaustible & 25 & 5 & 7 & 6 & 6 & 6 & 9 & 5 & 3 & 3 \\
\hline & $\begin{array}{c}\text { Easy to produce/manufacture/can } \\
\text { generate our own fuel/not } \\
\text { dependent on foreign sources }\end{array}$ & 18 & 3 & 7 & 6 & 1 & 1 & 7 & 3 & 3 & 2 \\
\hline & All other availability mentions & 15 & 3 & 3 & 3 & 4 & 3 & 5 & 2 & 4 & 3 \\
\hline \multirow{3}{*}{$\begin{array}{r}\text { Existing/developing } \\
\text { technology (net) }\end{array}$} & & 88 & 17 & 21 & 19 & 19 & 18 & 30 & 16 & 18 & 16 \\
\hline & $\begin{array}{l}\text { This technology is already being } \\
\text { developed/used }\end{array}$ & 34 & 6 & 8 & 7 & 7 & 7 & 12 & 6 & 6 & 6 \\
\hline & $\begin{array}{l}\text { Electric cars are already being } \\
\text { developed }\end{array}$ & 33 & 6 & 8 & 7 & 7 & 7 & 11 & 6 & 7 & 6 \\
\hline
\end{tabular}




\begin{tabular}{|c|c|c|c|c|c|c|c|c|c|c|c|}
\hline & \multirow{3}{*}{ Reasons } & \multicolumn{2}{|c|}{ Total } & \multicolumn{8}{|c|}{ Region } \\
\hline & & \multirow{2}{*}{$\mathbf{n}$} & \multirow{2}{*}{$\%$} & \multicolumn{2}{|c|}{$\mathbf{N E}^{1}$} & \multicolumn{2}{|c|}{$\mathrm{NC}^{1}$} & \multicolumn{2}{|c|}{$\mathbf{S}^{1}$} & \multicolumn{2}{|c|}{$\mathbf{W}^{1}$} \\
\hline & & & & $\mathbf{n}$ & $\%$ & $\mathbf{n}$ & $\%$ & $\mathbf{n}$ & $\%$ & $\mathbf{n}$ & $\%$ \\
\hline & $\begin{array}{l}\text { Many/more and more things are } \\
\text { powered by electric/all other } \\
\text { existing/ developing } \\
\text { technology mentions }\end{array}$ & 21 & 4 & 4 & 4 & 4 & 4 & 7 & 4 & 5 & 5 \\
\hline Economical/affordable & & 58 & 11 & 11 & 10 & 11 & 11 & 24 & 12 & 12 & 11 \\
\hline Methods of generating (net) & & 38 & 7 & 5 & 4 & 8 & 8 & 16 & 8 & 10 & 8 \\
\hline & Can be solar generated/powered & 26 & 5 & 2 & 2 & 6 & 5 & 10 & 5 & 8 & 7 \\
\hline & $\begin{array}{l}\text { All other methods of generating } \\
\text { mentions }\end{array}$ & 12 & 3 & 2 & 2 & 2 & 2 & 6 & 3 & 1 & 1 \\
\hline $\begin{array}{l}\text { Most familiar with it/not } \\
\text { familiar with others }\end{array}$ & & 38 & 7 & 9 & 8 & 10 & 10 & 12 & 6 & 7 & 6 \\
\hline Safety concerns (net) & & 26 & 5 & 8 & 7 & 3 & 3 & 10 & 5 & 4 & 4 \\
\hline $\begin{array}{l}\text { Best source } \\
\quad \text { (unspecified) }\end{array}$ & & 19 & 4 & 7 & 6 & 2 & 2 & 6 & 3 & 4 & 3 \\
\hline More efficient & & 17 & 3 & 3 & 3 & 4 & 4 & 4 & 2 & 6 & 5 \\
\hline $\begin{array}{l}\text { Easier/convenient } \\
\text { (unspecified) }\end{array}$ & & 16 & 3 & 6 & 6 & 2 & 2 & 7 & 4 & $\begin{array}{r}\text { small } \\
\text { base }\end{array}$ & $\begin{array}{r}\text { small } \\
\text { base }\end{array}$ \\
\hline $\begin{array}{l}\text { Others not practical } \\
\text { /performance concerns }\end{array}$ & & 15 & 3 & 3 & 3 & $\begin{array}{c}\text { small } \\
\text { base }\end{array}$ & $\begin{array}{c}\text { small } \\
\text { base }\end{array}$ & 6 & 3 & 5 & 5 \\
\hline Other & & 24 & 5 & 4 & 4 & 4 & 3 & 10 & 5 & 7 & 6 \\
\hline Don't know & & 34 & 6 & 4 & 4 & 10 & 10 & 15 & 8 & 4 & 3 \\
\hline
\end{tabular}

Source: ORCI for NREL (2000b), Study \#709489, N=522.

${ }^{1}$ In this report the following abbreviations stand for:

$\mathrm{NE}$ - Northeast region

$\mathrm{NC}$ - North-Central region

$\mathrm{S}-$ South Region

W - West Region 
Q4.1.5: Why did you say ethanol would be the best fuel for use in personal vehicles when gasoline is no longer available? (open-ended)

Table 4.1.5. Reasons Ethanol Would Be the Best Fuel for Use in Personal Vehicles When Gasoline Is No Longer Available

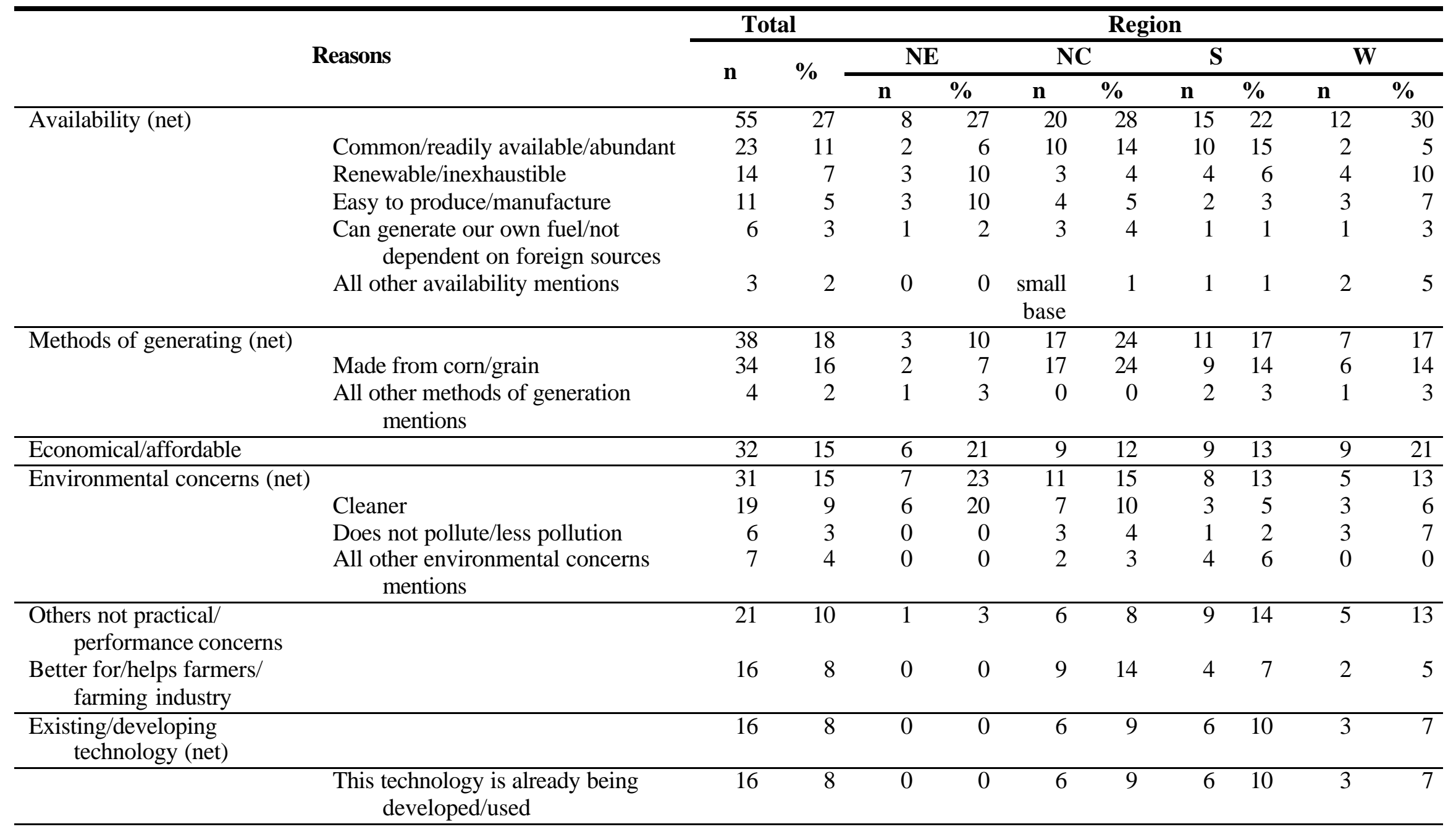




\begin{tabular}{|c|c|c|c|c|c|c|c|c|c|c|}
\hline \multirow{3}{*}{ Reasons } & \multicolumn{2}{|c|}{ Total } & \multicolumn{8}{|c|}{ Region } \\
\hline & \multirow{2}{*}{$\mathbf{n}$} & \multirow{2}{*}{$\%$} & \multicolumn{2}{|c|}{$\mathbf{N E}$} & \multicolumn{2}{|c|}{$\mathrm{NC}$} & \multicolumn{2}{|c|}{$\mathbf{S}$} & \multicolumn{2}{|c|}{$\mathbf{W}$} \\
\hline & & & $\mathbf{n}$ & $\%$ & $\mathbf{n}$ & $\%$ & $\mathbf{n}$ & $\%$ & $\mathbf{n}$ & $\%$ \\
\hline Best source (unspecified) & 11 & 5 & 2 & 5 & 4 & 6 & 1 & 2 & 4 & 10 \\
\hline More similar to gasoline & 10 & 5 & 3 & 9 & 3 & 4 & 2 & 3 & 2 & 5 \\
\hline Other & 25 & 12 & 8 & 24 & 4 & 6 & 8 & 13 & 5 & 13 \\
\hline Don't know & 15 & 7 & 0 & 0 & 5 & 7 & 8 & 13 & 1 & 3 \\
\hline
\end{tabular}

Source: ORCI for NREL (2000b), Study \#709489, N=206. 
Q4.1.6: Why did you say hydrogen would be the best fuel for use in personal vehicles when gasoline is no longer available? (open-ended)

Table 4.1.6. Reasons Hydrogen Would Be the Best Fuel for Use in Personal Vehicles When Gasoline Is No Longer Available

\begin{tabular}{|c|c|c|c|c|c|c|c|c|c|c|c|}
\hline \multirow{3}{*}{ Reasons } & & \multicolumn{2}{|c|}{ Total } & \multicolumn{8}{|c|}{ Region } \\
\hline & & \multirow[b]{2}{*}{$\mathbf{n}$} & \multirow[b]{2}{*}{$\%$} & \multicolumn{2}{|c|}{$\mathbf{N E}$} & \multicolumn{2}{|c|}{$\mathrm{NC}$} & \multicolumn{2}{|c|}{$\mathbf{S}$} & \multicolumn{2}{|c|}{$\mathbf{W}$} \\
\hline & & & & $\mathbf{n}$ & $\%$ & $\bar{n}$ & $\%$ & $\mathbf{n}$ & $\%$ & $\mathbf{n}$ & $\%$ \\
\hline \multirow[t]{5}{*}{ Availability (net) } & & 56 & 37 & 9 & 35 & 14 & 40 & 19 & 38 & 14 & 35 \\
\hline & $\begin{array}{l}\text { Common/readily } \\
\text { available/abundant }\end{array}$ & 41 & 27 & 8 & 30 & 10 & 31 & 14 & 28 & 9 & 21 \\
\hline & Easy to produce/manufacture & 10 & 7 & 2 & 6 & 5 & 14 & 2 & 4 & 2 & 5 \\
\hline & Renewable/inexhaustible & 7 & 5 & 0 & 0 & 0 & 0 & 4 & 8 & 3 & 8 \\
\hline & All other availability mentions & 5 & 3 & 1 & 5 & 2 & 4 & 0 & 0 & 3 & 5 \\
\hline \multirow{4}{*}{$\begin{array}{l}\text { Environmental concerns } \\
\quad \text { (net) }\end{array}$} & & 40 & 27 & 7 & 26 & 10 & 29 & 15 & 30 & 9 & 22 \\
\hline & Cleaner & 26 & 17 & 4 & 14 & 5 & 15 & 10 & 20 & 7 & 18 \\
\hline & Does not pollute/less pollution & 10 & 7 & 2 & 9 & 3 & 9 & 2 & 5 & 2 & 6 \\
\hline & $\begin{array}{l}\text { All other environmental concerns } \\
\text { mentions }\end{array}$ & 5 & 4 & 1 & 3 & 2 & 4 & 2 & 5 & 0 & 0 \\
\hline \multirow[t]{2}{*}{ Methods of generating (net) } & & 21 & 14 & 5 & 21 & 6 & 17 & 8 & 17 & 1 & 3 \\
\hline & $\begin{array}{l}\text { Can be generated by/derived from } \\
\text { water }\end{array}$ & 20 & 13 & 5 & 17 & 6 & 17 & 8 & 17 & 1 & 3 \\
\hline Economical/affordable & & 18 & 12 & 3 & 12 & 5 & 15 & 6 & 12 & 4 & 10 \\
\hline $\begin{array}{l}\text { Others not practical/ } \\
\text { performance concerns }\end{array}$ & & 17 & 11 & 5 & 19 & 4 & 13 & 4 & 8 & 3 & 8 \\
\hline \multirow{2}{*}{$\begin{array}{l}\text { Existing/developing } \\
\text { technology (net) }\end{array}$} & & 10 & 7 & 2 & 7 & 2 & 4 & 3 & 6 & 4 & 9 \\
\hline & $\begin{array}{l}\text { This technology is already being } \\
\text { developed/used }\end{array}$ & 10 & 7 & 2 & 7 & 2 & 4 & 3 & 6 & 4 & 9 \\
\hline
\end{tabular}




\begin{tabular}{|c|c|c|c|c|c|c|c|c|c|c|}
\hline More efficient & 8 & 5 & 1 & 5 & 0 & 0 & 3 & 7 & 3 & 8 \\
\hline Safety concerns & 8 & 5 & 1 & 5 & 1 & 2 & 1 & 3 & 4 & 10 \\
\hline Best source (unspecified) & 4 & 3 & 1 & 4 & $\begin{array}{c}\text { small } \\
\text { base }\end{array}$ & 1 & 1 & 2 & 2 & 4 \\
\hline Other & 13 & 9 & 1 & 5 & 4 & 13 & 2 & 4 & 6 & 14 \\
\hline Don't know & 13 & 9 & 1 & 4 & 1 & 3 & 8 & 15 & 4 & 9 \\
\hline
\end{tabular}

Source: ORCI for NREL (2000b), Study \#709489, N=151. 
Public Perceptions of Worst Fuel for Use in Personal Vehicles When Gasoline Is No Longer Available

Q4.1.7: Consider a future date when gasoline is no longer available. Which of the following do you think would be the worst fuel for use in personal vehicles: ethanol, hydrogen, or electricity?

Table 4.1.7. Public Perception of Worst Fuel for Use in Personal Vehicles When Gasoline Is No Longer Available

\begin{tabular}{lrc}
\hline $\begin{array}{l}\text { Worst Fuel for Use in Personal } \\
\text { Vehicles }\end{array}$ & Number & Percent \\
\hline Ethanol & 281 & 28 \\
Hydrogen & 274 & 27 \\
Electricity & 150 & 15 \\
Don't know & 295 & 30 \\
\hline Total & 1,000 & 100 \\
\hline
\end{tabular}

Source: ORCI for NREL (2000b), Study \#709489, N=1,000. 
Q4.1.8: Why did you say ethanol would be the worst fuel for use in personal vehicles when gasoline is no longer available? (openended)

Table 4.1.8. Reasons Ethanol Would Be the Worst Fuel for Use in Personal Vehicles When Gasoline Is No Longer Available

\begin{tabular}{|c|c|c|c|c|c|c|c|c|c|c|c|}
\hline & \multirow{3}{*}{ Reasons } & \multicolumn{2}{|c|}{ Total } & \multicolumn{8}{|c|}{ Region } \\
\hline & & \multirow{2}{*}{$\mathbf{n}$} & \multirow{2}{*}{$\%$} & \multicolumn{2}{|c|}{$\mathbf{N E}$} & \multicolumn{2}{|c|}{$\mathbf{N C}$} & \multicolumn{2}{|l|}{$\mathbf{S}$} & \multicolumn{2}{|c|}{$\bar{W}$} \\
\hline & & & & $\mathbf{n}$ & $\%$ & $\bar{n}$ & $\%$ & $\mathbf{n}$ & $\%$ & $\mathbf{n}$ & $\%$ \\
\hline \multirow{5}{*}{$\begin{array}{l}\text { Environmental concerns } \\
\quad \text { (net) }\end{array}$} & & 106 & 38 & 21 & 41 & 25 & 43 & 35 & 33 & 26 & 38 \\
\hline & Pollution (subnet) & 84 & 30 & 16 & 32 & 20 & 34 & 28 & 26 & 21 & 31 \\
\hline & Produces pollution & 46 & 16 & 9 & 18 & 9 & 16 & 21 & 20 & 7 & 10 \\
\hline & Causes air pollution & 27 & 10 & 5 & 10 & 7 & 12 & 5 & 5 & 9 & 14 \\
\hline & $\begin{array}{l}\text { All other environmental concerns } \\
\text { mentions }\end{array}$ & 17 & 6 & 3 & 7 & 4 & 6 & 5 & 5 & 6 & 8 \\
\hline \multirow[t]{4}{*}{ Safety concerns (net) } & & 56 & 20 & 17 & 34 & 12 & 21 & 17 & 17 & 10 & 14 \\
\hline & Flammable/combustible & 11 & 4 & 3 & 6 & 1 & 2 & 4 & 4 & 3 & 4 \\
\hline & Explosive & 7 & 3 & 2 & 4 & 1 & 2 & 4 & 4 & 0 & 0 \\
\hline & Contains chemicals & 7 & 3 & 3 & 7 & 1 & 2 & 1 & 1 & 2 & 3 \\
\hline Finite/exhaustible resource & & 8 & 3 & 2 & 4 & 0 & 0 & 2 & 2 & 3 & 5 \\
\hline Difficult to produce & & 8 & 3 & 1 & 2 & 2 & 3 & 3 & 3 & 1 & 2 \\
\hline Causes engine trouble & & 7 & 3 & 0 & 0 & 3 & 6 & 3 & 3 & 1 & 2 \\
\hline Other & & 38 & 13 & 4 & 10 & 9 & 16 & 11 & 11 & 12 & 20 \\
\hline Don't know & & 58 & 20 & 10 & 21 & 7 & 12 & 31 & 29 & 10 & 15 \\
\hline
\end{tabular}

Source: ORCI for NREL (2000b), Study \#709489, N=281. 
Q4.1.9: Why did you say hydrogen would be the worst fuel for use in personal vehicles when gasoline is no longer available? (open-ended)

Table 4.1.9. Reasons Hydrogen Would Be the Worst Fuel for Use in Personal Vehicles When Gasoline Is No Longer Available

\begin{tabular}{|c|c|c|c|c|c|c|c|c|c|c|c|}
\hline & \multirow{3}{*}{ Reasons } & \multicolumn{2}{|c|}{ Total } & \multicolumn{8}{|c|}{ Region } \\
\hline & & \multirow{2}{*}{$\mathbf{n}$} & \multirow{2}{*}{$\%$} & \multicolumn{2}{|c|}{$\mathbf{N E}$} & \multicolumn{2}{|c|}{$\mathrm{NC}$} & \multicolumn{2}{|c|}{$\mathbf{S}$} & \multicolumn{2}{|c|}{$\mathbf{W}$} \\
\hline & & & & $\mathbf{n}$ & $\%$ & $\mathbf{n}$ & $\%$ & $\mathbf{n}$ & $\%$ & $\mathbf{n}$ & $\%$ \\
\hline \multirow[t]{7}{*}{ Safety concerns (net) } & & 137 & 50 & 30 & 59 & 31 & 44 & 49 & 48 & 26 & 53 \\
\hline & Explosive & 39 & 14 & 9 & 17 & 6 & 9 & 17 & 17 & 7 & 15 \\
\hline & Flammable/combustible & 38 & 14 & 9 & 18 & 8 & 12 & 13 & 13 & 7 & 14 \\
\hline & Dangerous/not safe (unspecified) & 28 & 10 & 5 & 10 & 6 & 9 & 12 & 11 & 5 & 10 \\
\hline & Unstable & 14 & 5 & 4 & 8 & 3 & 5 & 2 & 2 & 4 & 8 \\
\hline & Think of bombs & 8 & 3 & 2 & 4 & 2 & 3 & 3 & 2 & 1 & 2 \\
\hline & All other safety concerns mentions & 24 & 9 & 2 & 4 & 10 & 14 & 5 & 5 & 5 & 11 \\
\hline $\begin{array}{l}\text { Pollution and environmental } \\
\text { concerns (net) }\end{array}$ & & 21 & 8 & 4 & 8 & 3 & 4 & 10 & 10 & 4 & 9 \\
\hline $\begin{array}{l}\text { Not enough is known about } \\
\text { it }\end{array}$ & & 10 & 4 & 1 & 2 & 3 & 5 & 3 & 3 & 3 & 6 \\
\hline Difficult to produce & & 10 & 4 & 2 & 5 & 3 & 4 & 5 & 5 & 0 & 0 \\
\hline Too expensive & & 8 & 3 & 0 & 0 & 2 & 2 & 4 & 4 & 2 & 4 \\
\hline Other & & 41 & 15 & 8 & 15 & 11 & 16 & 18 & 17 & 5 & 10 \\
\hline Don't know & & 57 & 21 & 9 & 17 & 20 & 28 & 19 & 19 & 9 & 18 \\
\hline
\end{tabular}

Source: ORCI for NREL (2000b), Study \#709489, N=274. 
Q4.1.10: Why did you say electricity would be the worst fuel for use in personal vehicles when gasoline is no longer available? (open-ended)

Table 4.1.10. Reasons Electricity Would Be the Worst Fuel for Use in Personal Vehicles When Gasoline Is No Longer Available

\begin{tabular}{|c|c|c|c|c|c|c|c|c|c|c|c|}
\hline & \multirow{3}{*}{ Reasons } & \multicolumn{2}{|c|}{ Total } & \multicolumn{8}{|c|}{ Region } \\
\hline & & \multirow{2}{*}{$\mathbf{n}$} & \multirow{2}{*}{$\%$} & \multicolumn{2}{|c|}{$\mathbf{N E}$} & \multicolumn{2}{|c|}{$\mathbf{N C}$} & \multicolumn{2}{|c|}{$\mathbf{S}$} & \multicolumn{2}{|c|}{$\mathbf{W}$} \\
\hline & & & & $\mathbf{n}$ & $\%$ & $\mathbf{n}$ & $\%$ & $\mathbf{n}$ & $\%$ & $\mathbf{n}$ & $\%$ \\
\hline Too Expensive & & 42 & 28 & 13 & 37 & 12 & 31 & 8 & 17 & 10 & 30 \\
\hline $\begin{array}{c}\text { Electric vehicles can't hold } \\
\text { charge for long/can't } \\
\text { travel long distances }\end{array}$ & & 30 & 20 & 4 & 11 & 9 & 23 & 11 & 25 & 6 & 19 \\
\hline \multirow{4}{*}{$\begin{array}{l}\text { Environmental concerns } \\
\quad \text { (net) }\end{array}$} & & 29 & 19 & 7 & 21 & 7 & 18 & 7 & 16 & 8 & 24 \\
\hline & $\begin{array}{l}\text { Must burn coal/fossil fuels to } \\
\text { generate electricity }\end{array}$ & 18 & 12 & 4 & 12 & 6 & 15 & 4 & 8 & 5 & 14 \\
\hline & Pollution & 8 & 6 & 4 & 11 & 2 & 6 & 2 & 6 & 0 & 0 \\
\hline & $\begin{array}{l}\text { All other environmental concerns } \\
\text { mentions }\end{array}$ & 9 & 6 & 1 & 4 & 0 & 0 & 5 & 10 & 3 & 10 \\
\hline Not enough electricity now & & 17 & 12 & 6 & 16 & 3 & 7 & 6 & 14 & 3 & 8 \\
\hline Safety concerns & & 8 & 5 & 1 & 3 & 2 & 5 & 3 & 6 & 2 & 7 \\
\hline Other & & 21 & 15 & 4 & 12 & 7 & 17 & 7 & 16 & 2 & 12 \\
\hline Don't know & & 15 & 10 & 3 & 8 & 4 & 10 & 6 & 13 & 3 & 8 \\
\hline
\end{tabular}

Source: ORCI for NREL (2000b), Study \#709489, N=150. 


\section{CONVENTIONAL, MORE FUEL-EFFICIENT, AND ADVANCED TECHNOLOGY VEHICLES}

Section 4 focuses on vehicle owners and the decisions they make about their vehicles. It consists of three sections that encompass survey data on owners' decisions about their conventional (i.e., gasoline) vehicles, as well as more fuel-efficient and advanced technology vehicles.

\subsection{VEHICLE OWNERS' DECISIONS ABOUT CONVENTIONAL VEHICLES}

Surveys revealed that one out of five of adults plan to keep his/her vehicle for 5 years (Table 5.1.1). On average, Americans expect to keep their vehicles for almost 7 years (Table 5.1.2). An overwhelming majority of Americans bought, rather than leased, their current vehicles, and almost one in two adults purchased their vehicles used. Only 7 percent of respondents reported leasing their current vehicles. Adults who leased vehicles tended to lease them new (Table 5.1.2). More than one out of four U.S. adults purchased their current vehicles in order to replace vehicles that had a lot of mileage, or ones that required expensive or frequent repairs (Table 5.1.3).

In 2001, Americans value vehicle safety as much as dependability, followed by vehicle quality, fuel economy, and low vehicle price (Table 5.1.4). In the year 2000, the public valued dependability more than safety, followed by quality. Over the years since 1981, the population reported dependability as the most important attribute in their choice of a new vehicle. Americans valued vehicle price after dependability from 1981 until 1987. Since 1996 safety became the second most important attribute (after dependability) when buying a new vehicle. With respect to safety, an overwhelming majority do not believe that a lighter vehicle is as safe in traffic accidents as a heavier one of the same size (Table 5.1.5).

In line with the fact that lately Americans consider safety one of the most valuable vehicle attributes and that the larger the vehicle the safer it is expected to be, is the fact that the highestselling vehicles in the United States currently are large vehicles such as pickup trucks, minivans, and sport utility vehicles (The Washington Post, 2001). In 1998, almost half of those vehicle owners who were likely to purchase the above-mentioned large vehicles planned on buying a towing package for the new vehic le (Table 5.1.6). In addition, about one-third of these vehicle owners planned on using their new pickup truck, minivan, standard van, or sport utility vehicle off-road (Table 5.1.7).

Despite the rising popularity of pickup trucks, vans, minivans, or sport utility vehicles, cars are also popular. In fact, according to the ORCI surveys, a majority of vehicle owners would most likely purchase cars rather than pickup trucks, vans, minivans or sport utility vehicles if they were to buy a new vehicle (Table 5.1.9). The vehicle owners who were likely to buy a car reported features and styles (such as comfort, smoothness and quietness of ride) that come with cars as a major reason of their preference of the car over the other vehicle types (Table 5.1.8).

When they dispose of their current vehicles, almost one out of two Americans will buy a new vehicle, and two out of five will buy a used vehicle (Table 5.1.9). This finding is consistent with survey results from 1998 when almost as many reported a preference for buying new vehicles as 
those who would rather purchase used ones (Table 5.1.10). In 1998, a majority of those Americans who were likely to purchase a new vehicle would plan on spending $\$ 10,000-$ $\$ 25,000$ on this new vehicle. More than one in four U.S. adults planned to spend $\$ 15,000-$ $\$ 20,000$ (Table 5.1.8). When asked about a dollar amount increase in new vehicle prices that would make them purchase a used vehicle instead of the new one, almost one in two adults mentioned more than $\$ 2,000$ (Table 5.1.10).

Q5.1.1: From the day you acquired the vehicle you currently drive, how many years total do you plan on keeping it (record number)?

Table 5.1.1. Total Number of Years Individuals Plan to Keep Their Vehicle

\begin{tabular}{lrrr}
\hline Number of Years & Number & Percent \\
\hline $1-5$ years (net) & 1 & 461 & 50 \\
& 2 & 35 & 4 \\
& 3 & 78 & 8 \\
& 4 & 97 & 11 \\
& 5 & 68 & 7 \\
$6-10$ & & 182 & 20 \\
$11-15$ & & 267 & 29 \\
$16-20$ & & 51 & 6 \\
Don't know & 51 & 6 \\
\hline Total & 91 & 10 \\
\hline Mean & & 920 & 101 \\
\hline
\end{tabular}

Source: ORCI for NREL (2000c), Study \#709089, N=920.

${ }^{1}$ In this report, calculation of means, medians and standard deviations are based on raw numbers. "Don't know" responses are not part of the calculations. 
Q5.1.2: How did you acquire the vehicle you currently drive: purchase, lease, gift?

Table 5.1.2. Vehicle Acquisition Data

\begin{tabular}{llcc}
\hline Acquisition Method & Number & Percent \\
\hline Purchased (net) & & 783 & 85 \\
& Purchased new & 333 & 36 \\
& Purchased used & 449 & 49 \\
Leased (net) & & 66 & 7 \\
& Leased new & 54 & 6 \\
Gift & Leased used & 12 & 1 \\
Other/don't know & & 60 & 7 \\
Total & & 12 & 1 \\
\hline Source: ORCl & & 920 & 100 \\
\hline
\end{tabular}

Source: ORCI for NREL (2000c), Study \#709089, N=920.

Q5.1.3: What was the primary reason you acquired the vehicle that you currently drive?

Table 5.1.3. Primary Reason for Acquiring Current Vehicle

\begin{tabular}{lrr}
\hline Primary Reason & Number & Percent \\
\hline $\begin{array}{l}\text { Replaced a vehicle that had a lot of mileage on it or one that } \\
\quad \text { required expensive or frequent repairs }\end{array}$ & 247 & 27 \\
Wanted a newer vehicle & 209 & 23 \\
Wanted/needed a different size or type of vehicle & 200 & 22 \\
Replaced a vehicle that was damaged in an accident or was & 79 & 9 \\
$\quad$ stolen & & \\
First vehicle & 63 & 7 \\
Reached end of previous lease & 25 & 3 \\
Other/don't know & 97 & 11 \\
\hline Total & 920 & 102 \\
\hline
\end{tabular}

Source: ORCI for NREL (2000c), Study \#709089, N=920. 
Q5.1.4: Which of the following attributes would be MOST important to you in your choice of your next vehicle?

Table 5.1.4. Trends in Vehicle Attribute Preference, Selected Years 1980-2001

\begin{tabular}{|c|c|c|c|c|c|c|c|c|c|c|c|c|}
\hline \multirow{2}{*}{ Attributes } & \multirow{2}{*}{$\begin{array}{c}1980 \\
(\%)\end{array}$} & \multirow{2}{*}{$\begin{array}{c}1981 \\
(\%)\end{array}$} & \multirow{2}{*}{$\begin{array}{c}1983 \\
(\%)\end{array}$} & \multirow{2}{*}{$\begin{array}{c}1985 \\
(\%)\end{array}$} & \multirow{2}{*}{$\begin{array}{c}1987 \\
(\%)\end{array}$} & \multirow{2}{*}{$\begin{array}{c}1996 \\
(\%)\end{array}$} & \multicolumn{2}{|c|}{1998} & \multicolumn{2}{|c|}{2000} & \multicolumn{2}{|c|}{2001} \\
\hline & & & & & & & $\mathbf{n}$ & $\%$ & $\mathbf{n}$ & $\%$ & $\mathbf{n}$ & $\%$ \\
\hline Fuel economy & 42 & 20 & 13 & 8 & 4 & 7 & 44 & 4 & 98 & 10 & 102 & 10 \\
\hline Dependability & 31 & 40 & 38 & 41 & 44 & 34 & 359 & 36 & 300 & 32 & 288 & 29 \\
\hline Low price & 14 & 21 & 30 & 29 & 31 & 11 & 53 & 5 & 104 & 11 & 81 & 8 \\
\hline Quality & 4 & 7 & 11 & 12 & 8 & 19 & 197 & 20 & 199 & 21 & 220 & 22 \\
\hline Safety & 9 & 12 & 9 & 10 & 14 & 29 & 334 & 34 & 222 & 24 & 291 & 29 \\
\hline $\begin{array}{l}\text { Don't know/ none } \\
\text { of these }\end{array}$ & & & & & & & 12 & 1 & 18 & 2 & 6 & 1 \\
\hline Total & 100 & 100 & 100 & 100 & 101 & 100 & 999 & 100 & 941 & 100 & 988 & 99 \\
\hline
\end{tabular}

Sources: For 1980s: J. D. Power (data based on new car buyers). For 1996: ORCI for NREL. For 1998: ORCI for NREL (1998a), N = 1,000. For 2000: ORCI for NREL (2000a), N=941. For 2001: ORCI for NREL (2001c), N = 989. 
Q5.1.5: Do you think that a lighter vehicle is as safe in traffic accidents as a heavier one of the same size?

Table 5.1.5. Public Perception of Vehicle Size vs. Safety

\begin{tabular}{|c|c|c|c|c|c|c|c|c|c|c|c|c|}
\hline \multirow{3}{*}{ Vehicle Size as a Safety Issue } & \multicolumn{2}{|c|}{ Total } & \multicolumn{10}{|c|}{ Type of New Vehicle Will Purchase Next } \\
\hline & \multirow{2}{*}{$\mathbf{n}$} & \multirow{2}{*}{$\%$} & \multicolumn{2}{|c|}{ Small Car } & \multicolumn{2}{|c|}{$\begin{array}{c}\text { Large } \\
\text { Car }\end{array}$} & \multicolumn{2}{|c|}{ Minivan } & \multicolumn{2}{|c|}{ SUV } & \multicolumn{2}{|c|}{$\begin{array}{c}\text { Pickup } \\
\text { truck/van }\end{array}$} \\
\hline & & & $\mathbf{n}$ & $\%$ & $\mathbf{n}$ & $\%$ & $\mathbf{n}$ & $\%$ & $\mathbf{n}$ & $\%$ & $\mathbf{n}$ & $\%$ \\
\hline $\begin{array}{l}\text { Believe that a lighter vehicle is as safe } \\
\text { in traffic accidents as a heavier one } \\
\text { of the same size. }\end{array}$ & 119 & 12 & 23 & 15 & 16 & 7 & 13 & 19 & 27 & 15 & 24 & 13 \\
\hline $\begin{array}{l}\text { Do not believe that a lighter vehicle is } \\
\text { as safe in traffic accidents as a } \\
\text { heavier one of the same size }\end{array}$ & 824 & 82 & 127 & 81 & 193 & 89 & 52 & 75 & 136 & 78 & 156 & 82 \\
\hline Don't know & 57 & 6 & 8 & 5 & 7 & 3 & 4 & 6 & 11 & 6 & 10 & 5 \\
\hline Total & 1000 & 100 & 158 & 101 & 216 & 99 & 69 & 100 & 174 & 99 & 190 & 100 \\
\hline
\end{tabular}

Source: ORCI for NREL (1999b), Study \#70844, N=1,000. 
Q5.1.6: You mentioned that you would plan to buy a pickup truck, minivan, standard van, or sport utility vehicle. Would you plan to purchase a towing package for this new vehicle?

Table 5.1.6. Towing Package Preference for a New Vehicle

\begin{tabular}{|c|c|c|c|c|c|c|c|c|c|c|c|c|c|c|c|c|}
\hline \multirow{3}{*}{$\begin{array}{l}\text { Plans on } \\
\text { Purchasing } \\
\text { or Not } \\
\text { Purchasing } \\
\text { a Towing } \\
\text { Package }\end{array}$} & \multicolumn{6}{|c|}{ Total } & \multicolumn{10}{|c|}{ Type of New Vehicle Most Likely to Buy } \\
\hline & \multirow{2}{*}{$\mathbf{n}$} & \multirow{2}{*}{$\%$} & \multicolumn{2}{|c|}{$\begin{array}{c}\text { Small } \\
\text { Car }\end{array}$} & \multicolumn{2}{|c|}{$\begin{array}{l}\text { Midsized } \\
\text { Car }\end{array}$} & \multicolumn{2}{|c|}{ Large Car } & \multicolumn{2}{|c|}{$\begin{array}{c}\text { Pickup } \\
\text { truck }\end{array}$} & \multicolumn{2}{|c|}{$\begin{array}{c}\text { Standard } \\
\text { Van }\end{array}$} & \multicolumn{2}{|c|}{ Minivan } & \multicolumn{2}{|c|}{ SUV } \\
\hline & & & $\mathbf{n}$ & $\%$ & $\mathbf{n}$ & $\%$ & $\mathbf{n}$ & $\%$ & $\mathbf{n}$ & $\%$ & $\mathbf{n}$ & $\%$ & $\mathbf{n}$ & $\%$ & $\mathbf{n}$ & $\%$ \\
\hline $\begin{array}{l}\text { Plan on } \\
\text { buying a } \\
\text { towing } \\
\text { package }\end{array}$ & 208 & 47 & 0 & $\overline{0}$ & $\overline{0}$ & $\overline{0}$ & 0 & 0 & 85 & 52 & 6 & 33 & 25 & 32 & 91 & 51 \\
\hline $\begin{array}{l}\text { Do not plan } \\
\text { on buying } \\
\text { a towing } \\
\text { package }\end{array}$ & 225 & 51 & 0 & 0 & 0 & 0 & 0 & 0 & 77 & 47 & 13 & 67 & 52 & 66 & 83 & 47 \\
\hline Don't know & 6 & 1 & 0 & 0 & 0 & 0 & 0 & 0 & 2 & 1 & 0 & 0 & 1 & 2 & 3 & 2 \\
\hline Total & 439 & 99 & 0 & 0 & 0 & 0 & 0 & 0 & 164 & 100 & 19 & 100 & 78 & 100 & 177 & 100 \\
\hline
\end{tabular}

Source: ORCI for NREL (1998a), Study \#707089, N=439. 
Q5.1.7: You mentioned that you would plan to buy a pickup truck, minivan, standard van, or sport utility vehicle. Would you plan to use it off road?

Table 5.1.7. Expected Off-Road Use of a New Vehicle

\begin{tabular}{|c|c|c|c|c|c|c|c|c|c|c|c|c|c|c|c|c|}
\hline \multirow{3}{*}{$\begin{array}{l}\text { Plans on } \\
\text { Using Or } \\
\text { Not Using } \\
\text { Off-Road }\end{array}$} & \multicolumn{2}{|c|}{ Total } & \multicolumn{14}{|c|}{ Type of New Vehicle Most Likely to Buy } \\
\hline & \multirow{2}{*}{$\mathbf{n}$} & \multirow{2}{*}{$\%$} & \multicolumn{2}{|c|}{ Small Car } & \multicolumn{2}{|c|}{$\begin{array}{l}\text { Midsized } \\
\text { Car }\end{array}$} & \multicolumn{2}{|c|}{ Large Car } & \multicolumn{2}{|c|}{$\begin{array}{c}\text { Pickup } \\
\text { truck }\end{array}$} & \multicolumn{2}{|c|}{$\begin{array}{c}\text { Standard } \\
\text { Van }\end{array}$} & \multicolumn{2}{|c|}{ Minivan } & \multicolumn{2}{|c|}{ SUV } \\
\hline & & & $\mathbf{n}$ & $\%$ & $\mathbf{n}$ & $\%$ & $\mathbf{n}$ & $\%$ & $\mathbf{n}$ & $\%$ & $\mathbf{n}$ & $\%$ & $\mathbf{n}$ & $\%$ & $\mathbf{n}$ & $\%$ \\
\hline $\begin{array}{l}\text { Plan on } \\
\text { using off } \\
\text { road }\end{array}$ & 163 & 37 & 0 & 0 & 0 & $\overline{0}$ & 0 & 0 & 77 & 47 & 3 & 16 & 6 & 8 & 76 & 43 \\
\hline $\begin{array}{c}\text { Do not plan } \\
\text { on using } \\
\text { off road }\end{array}$ & 270 & 62 & 0 & 0 & 0 & 0 & 0 & 0 & 85 & 52 & 16 & 84 & 71 & 91 & 97 & 55 \\
\hline $\begin{array}{l}\text { Don't } \\
\text { know }\end{array}$ & 6 & 1 & 0 & 0 & 0 & 0 & 0 & 0 & 2 & 1 & 0 & 0 & 1 & 1 & 4 & 2 \\
\hline Total & 439 & 100 & 0 & 0 & 0 & 0 & 0 & 0 & 164 & 100 & 18 & 100 & 78 & 100 & 177 & 100 \\
\hline
\end{tabular}

Source: ORCI for NREL (1998a), Study \#707089, N=439. 
Q5.1.8: How much would you plan on paying for a new vehicle?

Table 5.1.8. Expected Expenditure for a New Vehicle

\begin{tabular}{|c|c|c|c|c|c|c|c|c|c|c|c|c|c|c|c|c|}
\hline \multirow{3}{*}{$\begin{array}{l}\text { Expected } \\
\text { Expenditure for } \\
\text { a New Vehicle }\end{array}$} & \multicolumn{2}{|c|}{ Total } & \multicolumn{14}{|c|}{ Type of New Vehicle Most likely to Buy } \\
\hline & \multirow{2}{*}{$\mathbf{n}$} & \multirow{2}{*}{$\%$} & \multicolumn{2}{|c|}{ Small Car } & \multicolumn{2}{|c|}{ Midsized Car } & \multicolumn{2}{|c|}{ Large Car } & \multicolumn{2}{|c|}{ Pickup truck } & \multicolumn{2}{|c|}{ Standard Van } & \multicolumn{2}{|c|}{ Minivan } & \multicolumn{2}{|l|}{ SUV } \\
\hline & & & $\mathbf{n}$ & $\%$ & $\mathbf{n}$ & $\%$ & $\mathbf{n}$ & $\%$ & $\mathbf{n}$ & $\%$ & $\mathbf{n}$ & $\%$ & $\mathbf{n}$ & $\%$ & $\mathbf{n}$ & $\%$ \\
\hline$\$ 5,000$ or less & 35 & 4 & 5 & 5 & 13 & 4 & 4 & 4 & 4 & 3 & 1 & 6 & 4 & 6 & 3 & 1 \\
\hline$\$ 5,001-\$ 10,000$ & 61 & 6 & 17 & 18 & 28 & 8 & 5 & 5 & 5 & 3 & 1 & 6 & 3 & 4 & 1 & 1 \\
\hline$\$ 10,001-\$ 15,000$ & 179 & 19 & 36 & 39 & 89 & 27 & 1 & 1 & 30 & 19 & 3 & 16 & 10 & 12 & 10 & 6 \\
\hline$\$ 15,001-\$ 20,000$ & 259 & 27 & 21 & 23 & 102 & 31 & 11 & 12 & 56 & 34 & 7 & 35 & 30 & 38 & 32 & 18 \\
\hline$\$ 20,001-\$ 25,000$ & 178 & 19 & 5 & 5 & 49 & 15 & 23 & 24 & 34 & 21 & 2 & 11 & 16 & 20 & 50 & 28 \\
\hline$\$ 25,001-\$ 30,000$ & 127 & 13 & 2 & 2 & 19 & 6 & 20 & 21 & 22 & 13 & 2 & 13 & 11 & 14 & 52 & 30 \\
\hline $\begin{array}{c}\text { More than } \\
\$ 30,000\end{array}$ & 70 & 7 & 1 & 1 & 11 & 3 & 26 & 27 & 7 & 4 & 3 & 13 & 2 & 2 & 21 & 12 \\
\hline Don't know & 50 & 5 & 7 & 7 & 23 & 7 & 4 & 4 & 5 & 3 & 0 & 0 & 3 & 4 & 8 & 4 \\
\hline Total & 959 & 100 & 94 & 100 & 334 & 101 & 94 & 98 & 163 & 100 & 19 & 100 & 79 & 100 & 177 & 100 \\
\hline Mean $^{1}$ & 20,650 & & 14,494 & & 18,022 & & 27,513 & & 20,427 & & 21,003 & & 19,487 & & 25,662 & \\
\hline
\end{tabular}

Source: ORCI for NREL (1998a), Study \#707089, N=959.

${ }^{1}$ In this report, calculation of means, medians and standard deviations are based on raw numbers. "Don't know" responses are not part of the calculations. 
Q5.1.9: If you were to buy a car, what is the major reason you prefer a car to a pickup truck, minivan, standard van, or sport utility vehicle? (open-ended)

Table 5.1.9. Major Reasons for Preferring a Car to Other Vehicle Types

\begin{tabular}{|c|c|c|c|c|c|c|c|c|c|c|c|c|c|c|c|c|c|c|c|c|c|}
\hline \multirow{3}{*}{ Reasons } & & \multicolumn{2}{|c|}{ Total } & \multicolumn{4}{|c|}{ Next Vehicle Purchase } & \multicolumn{14}{|c|}{ Type of New Vehicle Most Likely to Buy } \\
\hline & & \multirow[t]{2}{*}{$\mathbf{n}$} & \multirow[t]{2}{*}{$\%$} & \multicolumn{2}{|c|}{ New } & \multicolumn{2}{|c|}{ Used } & \multicolumn{2}{|c|}{$\begin{array}{l}\text { Small } \\
\text { Car }\end{array}$} & \multicolumn{2}{|c|}{$\begin{array}{l}\text { Midsized } \\
\text { Car }\end{array}$} & \multicolumn{2}{|c|}{$\begin{array}{l}\text { Large } \\
\text { Car }\end{array}$} & \multicolumn{2}{|c|}{$\begin{array}{l}\text { Pickup } \\
\text { Truck }\end{array}$} & \multicolumn{2}{|c|}{$\begin{array}{l}\text { Standard } \\
\text { Van }\end{array}$} & \multicolumn{2}{|c|}{$\begin{array}{c}\text { Mini } \\
\text { van }\end{array}$} & \multicolumn{2}{|c|}{ SUV } \\
\hline & & & & $\mathbf{n}$ & $\%$ & $\mathbf{n}$ & $\%$ & $\mathbf{n}$ & $\%$ & $\mathbf{n}$ & $\%$ & $\mathbf{n}$ & $\%$ & $\mathbf{n}$ & $\%$ & $\mathbf{n}$ & $\%$ & $\mathbf{n}$ & $\%$ & $\mathbf{n}$ & $\%$ \\
\hline $\begin{array}{r}\text { Features/styles/ } \\
\text { options (net) }\end{array}$ & & 376 & 72 & 180 & 74 & 157 & 70 & 68 & 73 & 238 & 71 & 70 & 75 & 0 & 0 & 0 & 0 & 0 & 0 & 0 & 0 \\
\hline \multirow[t]{5}{*}{ Performance (subnet) } & & 170 & 33 & 81 & 33 & 71 & 31 & 46 & 49 & 103 & 31 & 21 & 23 & 0 & 0 & 0 & 0 & 0 & 0 & 0 & 0 \\
\hline & $\begin{array}{l}\text { Size options/car } \\
\text { smaller/larger }\end{array}$ & 91 & 18 & 46 & 19 & 34 & 15 & 30 & 33 & 49 & 15 & 12 & 13 & 0 & 0 & 0 & 0 & 0 & 0 & 0 & 0 \\
\hline & $\begin{array}{l}\text { Easy to handle/ease } \\
\text { of } \\
\text { maneuverability }\end{array}$ & 76 & 15 & 33 & 14 & 36 & 16 & 23 & 25 & 49 & 15 & 3 & 4 & 0 & 0 & 0 & 0 & 0 & 0 & 0 & 0 \\
\hline & Smooth/quiet ride & 22 & 4 & 8 & 3 & 11 & 5 & 1 & 1 & 14 & 4 & 7 & 7 & 0 & 0 & 0 & 0 & 0 & 0 & 0 & 0 \\
\hline & $\begin{array}{c}\text { Stronger } \\
\text { horsepower/faster/ } \\
\text { better acceleration }\end{array}$ & 10 & 2 & 5 & 2 & 4 & 2 & 3 & 4 & 6 & 2 & 1 & 1 & 0 & 0 & 0 & 0 & 0 & 0 & 0 & 0 \\
\hline \multirow[t]{4}{*}{ Interior (subet) } & & 140 & 27 & 66 & 27 & 62 & 28 & 13 & 14 & 88 & 26 & 39 & 42 & 0 & 0 & 0 & 0 & 0 & 0 & 0 & 0 \\
\hline & Comfort & 82 & 16 & 45 & 18 & 34 & 15 & 8 & 8 & 41 & 12 & 33 & 36 & 0 & 0 & 0 & 0 & 0 & 0 & 0 & 0 \\
\hline & $\begin{array}{l}\text { Roomier/capacity to } \\
\text { hold more } \\
\text { passengers }\end{array}$ & 51 & 10 & 19 & 8 & 28 & 12 & 4 & 5 & 38 & 11 & 8 & 9 & & & 0 & 0 & 0 & 0 & 0 & 0 \\
\hline & Easier to get into & 23 & 4 & 7 & 3 & 11 & 5 & 1 & 1 & 19 & 6 & 3 & 3 & & & 0 & 0 & 0 & 0 & 0 & 0 \\
\hline $\begin{array}{l}\text { Don't need/use } \\
\text { features/styles/ } \\
\text { options offered on } \\
\text { pickup truck/ } \\
\text { minivan/ standard } \\
\text { van/sport utility } \\
\text { vehicle }\end{array}$ & & 67 & 13 & 26 & 11 & 27 & 12 & 7 & 7 & 48 & 14 & 13 & 13 & & & 0 & 0 & 0 & 0 & 0 & 0 \\
\hline
\end{tabular}




\begin{tabular}{|c|c|c|c|c|c|c|c|c|c|c|c|c|c|c|c|c|c|c|c|c|c|}
\hline \multirow{3}{*}{ Reasons } & & \multicolumn{2}{|c|}{ Total } & \multicolumn{4}{|c|}{ Next Vehicle Purchase } & \multicolumn{14}{|c|}{ Type of New Vehicle Most Likely to Buy } \\
\hline & & \multirow[t]{2}{*}{$\mathbf{n}$} & \multirow[t]{2}{*}{$\%$} & \multicolumn{2}{|c|}{ New } & \multicolumn{2}{|c|}{ Used } & \multicolumn{2}{|c|}{$\begin{array}{l}\text { Small } \\
\text { Car }\end{array}$} & \multicolumn{2}{|c|}{$\begin{array}{l}\text { Midsized } \\
\text { Car }\end{array}$} & \multicolumn{2}{|c|}{$\begin{array}{l}\text { Large } \\
\text { Car }\end{array}$} & \multicolumn{2}{|c|}{$\begin{array}{l}\text { Pickup } \\
\text { Truck }\end{array}$} & \multicolumn{2}{|c|}{$\begin{array}{c}\text { Standard } \\
\text { Van }\end{array}$} & \multicolumn{2}{|c|}{$\begin{array}{c}\text { Mini } \\
\text { van }\end{array}$} & \multicolumn{2}{|c|}{ SUV } \\
\hline & & & & $\mathbf{n}$ & $\%$ & $\mathbf{n}$ & $\%$ & $\mathbf{n}$ & $\%$ & $\mathbf{n}$ & $\%$ & $\mathbf{n}$ & $\%$ & $\mathbf{n}$ & $\%$ & $\mathbf{n}$ & $\%$ & $\mathbf{n}$ & $\%$ & $\mathbf{n}$ & $\%$ \\
\hline $\begin{array}{c}\text { Safety features/better } \\
\text { safety record }\end{array}$ & & 30 & 6 & 17 & 7 & 12 & 5 & 2 & 2 & 14 & 4 & 14 & 14 & & & 0 & 0 & 0 & 0 & 0 & 0 \\
\hline $\begin{array}{l}\text { Prefer car for other } \\
\text { features/styles/ } \\
\text { options }\end{array}$ & & 43 & 8 & 26 & 11 & 13 & 6 & 8 & 8 & 26 & 7 & 8 & 10 & & & 0 & 0 & 0 & 0 & 0 & 0 \\
\hline Need a "family car" & & 29 & 6 & 11 & 4 & 17 & 7 & 3 & 3 & 22 & 7 & 4 & 4 & 0 & 0 & 0 & 0 & 0 & 0 & 0 & 0 \\
\hline \multirow[t]{4}{*}{ Cost savings (net) } & & 98 & 19 & 41 & 17 & 49 & 22 & 36 & 39 & 56 & 17 & 7 & 7 & 0 & 0 & 0 & 0 & 0 & 0 & 0 & 0 \\
\hline & $\begin{array}{l}\text { Better gas } \\
\text { mileage/fuel } \\
\text { efficiency }\end{array}$ & 70 & 13 & 28 & 12 & 34 & 15 & 28 & 31 & 38 & 11 & 3 & 3 & 0 & 0 & 0 & 0 & 0 & 0 & 0 & 0 \\
\hline & Lower sticker price & 16 & 3 & 5 & 2 & 11 & 5 & 4 & 4 & 12 & 4 & 0 & 0 & 0 & 0 & 0 & 0 & 0 & 0 & 0 & 0 \\
\hline & $\begin{array}{l}\text { Less expensive to } \\
\text { operate/ lower } \\
\text { maintenance costs }\end{array}$ & 20 & 3 & 9 & 3 & 10 & 4 & 6 & 7 & 8 & 2 & 4 & 5 & 0 & 0 & 0 & 0 & 0 & 0 & 0 & 0 \\
\hline $\begin{array}{l}\text { Own a } \\
\text { truck/minivan/stan } \\
\text { dard van/sport } \\
\text { utility vehicle }\end{array}$ & & 38 & 7 & 20 & 8 & 15 & 7 & 5 & 5 & 23 & 7 & 10 & 11 & 0 & 0 & 0 & 0 & 0 & 0 & 0 & 0 \\
\hline $\begin{array}{l}\text { Prefer car/just don't } \\
\text { like/want pickup } \\
\text { truck/minivan/stan } \\
\text { dard van/sport } \\
\text { utility vehicle } \\
\end{array}$ & & 31 & 6 & 14 & 6 & 14 & 6 & 2 & 2 & 22 & 7 & 7 & 8 & 0 & 0 & 0 & 0 & 0 & 0 & 0 & 0 \\
\hline Car is more convenient & & 15 & 3 & 5 & 2 & 7 & 3 & 5 & 5 & 8 & 2 & 2 & 2 & 0 & 0 & 0 & 0 & 0 & 0 & 0 & 0 \\
\hline $\begin{array}{l}\text { More experience with } \\
\text { driving a car }\end{array}$ & & 13 & 3 & 4 & 2 & 7 & 3 & 2 & 2 & 7 & 2 & 2 & 2 & 0 & 0 & 0 & 0 & 0 & 0 & 0 & 0 \\
\hline Other & & 14 & 3 & 6 & 2 & 6 & 3 & 5 & 5 & 6 & 2 & 3 & 3 & 0 & 0 & 0 & 0 & 0 & 0 & 0 & 0 \\
\hline Don't know/no reason & & 14 & 3 & 6 & 2 & 6 & 3 & 1 & 1 & 12 & 4 & 1 & 2 & 0 & 0 & 0 & 0 & 0 & 0 & 0 & 0 \\
\hline
\end{tabular}

Source: ORCI for NREL (1998a), Study \#707089, N=520 
Q5.1.10: When you dispose of your current vehicle, how will you most likely replace it: buy a new vehicle, buy a used vehicle, lease a new vehicle, or lease a used vehicle?

Table 5.1.10. Current Vehicle Replacement Methods

\begin{tabular}{|c|c|c|c|c|}
\hline \multicolumn{3}{|c|}{ Method to Replace Vehicle } & \multirow{2}{*}{$\begin{array}{c}\text { Number } \\
819\end{array}$} & \multirow{2}{*}{$\begin{array}{r}\text { Percent } \\
89\end{array}$} \\
\hline Any (net) & & & & \\
\hline & $\begin{array}{l}\text { Buy } \\
\text { (subnet) }\end{array}$ & & 773 & 84 \\
\hline & & Buy a new vehicle & 422 & 46 \\
\hline & & Buy a used vehicle & 351 & 38 \\
\hline & $\begin{array}{l}\text { Lease } \\
\text { (subnet) }\end{array}$ & & 46 & 5 \\
\hline & & Lease a new vehicle & 42 & 5 \\
\hline & & Lease a used vehicle & 4 & $\begin{array}{l}\text { small } \\
\text { base }\end{array}$ \\
\hline Won't replace It & & & 57 & 6 \\
\hline $\begin{array}{l}\text { Receive a donated } \\
\text { vehicle/gift/other/ } \\
\text { don't know }\end{array}$ & & & 45 & 4 \\
\hline Total & & & 921 & 99 \\
\hline
\end{tabular}

Source: ORCI for NREL (2000c), Study \#709089, N=920 
Q5.1.11: Will the next vehicle you purchase be new or used?

Table 5.1.11. Public Preference for Purchasing New or Used Vehicles

\begin{tabular}{|c|c|c|c|c|c|c|c|c|c|c|c|c|c|c|c|c|}
\hline \multirow{3}{*}{$\begin{array}{l}\text { Type of } \\
\text { Vehicle }\end{array}$} & \multicolumn{2}{|c|}{ Total } & \multicolumn{14}{|c|}{ Type of New Vehicle Most likely to Buy } \\
\hline & \multirow{2}{*}{$\mathbf{n}$} & \multirow{2}{*}{$\%$} & \multicolumn{2}{|c|}{$\begin{array}{c}\text { Small } \\
\text { Car }\end{array}$} & \multicolumn{2}{|c|}{$\begin{array}{l}\text { Midsized } \\
\text { Car }\end{array}$} & \multicolumn{2}{|c|}{ Large Car } & \multicolumn{2}{|c|}{ Pickup truck } & \multicolumn{2}{|c|}{$\begin{array}{l}\text { Standard } \\
\text { Van }\end{array}$} & \multicolumn{2}{|c|}{ Minivan } & \multicolumn{2}{|c|}{ SUV } \\
\hline & & & $\mathbf{n}$ & $\%$ & $\mathbf{n}$ & $\%$ & $\mathbf{n}$ & $\%$ & $\mathbf{n}$ & $\%$ & $\mathbf{n}$ & $\%$ & $\mathbf{n}$ & $\%$ & $\mathbf{n}$ & $\%$ \\
\hline New vehicle & 452 & 45 & 41 & 44 & 158 & 47 & 45 & 48 & 64 & 39 & 9 & 46 & 37 & 46 & 98 & 55 \\
\hline Used vehicle & 456 & 46 & 43 & 46 & 147 & 44 & 35 & 38 & 92 & 56 & 10 & 54 & 39 & 50 & 68 & 39 \\
\hline $\begin{array}{c}\text { Don't plan to } \\
\text { purchase } \\
\text { vehicle }\end{array}$ & 49 & 5 & 6 & 6 & 19 & 6 & 9 & 10 & 3 & 2 & 0 & 0 & 2 & 3 & 1 & 1 \\
\hline Don't know & 43 & 4 & 3 & 4 & 10 & 3 & 5 & 5 & 6 & 3 & 0 & 0 & 1 & 1 & 9 & 5 \\
\hline Total & 1,000 & 100 & 93 & 100 & 334 & 100 & 94 & 101 & 165 & 100 & 19 & 100 & 79 & 100 & 176 & 100 \\
\hline
\end{tabular}

Source: ORCI for NREL (1998a), Study \#707089, N=1,000. 
Q5.1.12: Assuming energy or environmental concerns cause new vehicle prices to increase, how much would new vehicle prices have to increase for you to decide to buy a used vehicle instead of a new vehicle? (open-ended)

Table 5.1.12. Public Perception of the Effect of New Vehicle Price Increases on Purchasing Decisions

\begin{tabular}{lrr}
\hline $\begin{array}{l}\text { Amount Vehicle Prices Would Have to Increase Before Decision } \\
\text { is Made to Buy a Used Vehicle Instead of a New Vehicle }\end{array}$ & Number & Percent \\
\hline$\$ 500$ or less & 38 & 4 \\
$\$ 501-\$ 1,000$ & 45 & 5 \\
$\$ 1,001-\$ 2,000$ & 84 & 8 \\
$\$ 2,001-\$ 5,000$ & 270 & 27 \\
More than $\$ 5,000$ & 214 & 21 \\
None & 164 & 16 \\
Don't Know & 185 & 19 \\
\hline Total & 1,000 & 100 \\
\hline Mean' (including none) & 4,109 \\
\hline Mean' (excluding none) & 5,142 \\
\hline
\end{tabular}

Source: ORCI for NREL (1998a), Study \#707089, N=1,000.

${ }^{1}$ In this report, calculation of means, medians and standard deviations are based on raw numbers. "Don't know" responses are not part of the calculations.

\subsection{VEHICLE OWNERS' DECISIONS ABOUT MORE FUEL-EFFICIENT VEHICLES}

Though one out of four Americans did not consider fuel economy an important issue when purchasing his/her current vehicle (Table 5.2.1), the ORCI surveys revealed that one out of two Americans is interested in having more information about the environmental impacts of new light vehicles (Table 5.2.2).

A relatively high percentage of the U.S. population who does not consider fuel economy an important issue is consistent with relatively low willingness to purchase a car with better fuel economy. When buying a new vehicle, slightly more than one in four Americans would most likely purchase a lighter car with average acceleration and better fuel economy rather than an average car, i.e., a car with average weight, average acceleration and average fuel economy (Table 5.2.3). However, respondents gave their highest preference to the lighter car with average acceleration and better fuel economy over four other choices.

Among the things that would motivate purchasing a more fuelefficient vehicle, American adults mentioned cost, features and performance, and gas mileage (Table 5.2.4). With cost being a critical factor in motivating them to purchase a more fuelefficient vehicle, on average, Americans say they would pay more than $\$ 2,100$ additional for a vehicle that gets 10 percent better fuel economy (Table 5.2.5). 
Consistent with the finding that one out of two U.S. adults is interested in having more information on the environmental impacts of new light vehicles, the other ORCI survey found that slightly more than half of Americans would rather purchase a new vehicle with a more fuelefficient 'optional' engine, given that fuel supply can be conveniently purchased. The survey defined an 'optional' engine as the engine requiring new fuel that costs the same as gasoline and is just as good as gasoline. According to the survey, the U.S. public would purchase the more fuelefficient engine if fuel supply were available at one in three stations (Table 5.2.6).

Though Americans would make their decisions on purchasing or not purchasing more fuel efficient vehicles based on cost, performance, and other considerations rather than on reports presenting proof of fuel efficiency, more than half of U.S. adults had some information or paid some attention to the information on fuel economy when purchasing their current vehicle. They reported finding this information on window stickers, in dealer brochures, magazines, and consumer guides. Though Americans were more likely to get information on fuel economy from sources other than the Government's Fuel Economy Guide, more than one in five respondents mentioned seeing a copy of it. Most of them had seen it in a showroom (Table 5.2.7).

Q5.2.1: If fuel economy was a factor you considered in your last vehicle purchase, where did you find information on fuel economy?

Table 5.2.1. Location of Fuel Economy Data Used for Last Vehicle Purchase

\begin{tabular}{llrr}
\hline Location of Fuel Economy Information & Number & Percent \\
\hline Any (net) & Window sticker & 561 & 56 \\
& Dealer brochures & 222 & 22 \\
& Magazines, consumer guide & 116 & 12 \\
& Word of mouth & 113 & 11 \\
& On the Internet & 50 & 5 \\
& Television & 39 & 4 \\
& Government's Fuel Economy & 23 & 2 \\
& Guide & 6 & 1 \\
& Other & 79 & 8 \\
\hline Fuel economy not an & & 262 & \\
important issue & & 177 \\
\hline Don't know/don't own & & 1,000 & 100 \\
\hline vehicle & & & \\
\hline Total & & & \\
\hline
\end{tabular}

Source: ORCI for NREL (1999a), Study \#70809, N=1,000. 
Q5.2.2: On a scale of 1 to 5 , where 1 is not interested and 5 is very interested, please indicate your interest in having more information about the environmental impacts of new cars and light trucks?

Table 5.2.2. Public Interest for Additional Information on the Environmental Impacts of New Light Vehicles

\begin{tabular}{lrr}
\hline \multicolumn{1}{c}{ Level of Interest } & Number & Percent \\
\hline Very interested & 230 & 23 \\
Moderately interested & 143 & 14 \\
Somewhat interested & 254 & 25 \\
Little interested & 92 & 9 \\
Not interested & 265 & 27 \\
Don't know & 16 & 2 \\
\hline Total & 1,000 & 100 \\
\hline
\end{tabular}

Source: ORCI for NREL (1999a), Study \#70809, N=1,000.

Q5.2.3: Some of the things that are important in choosing a new car are power, acceleration time, and fuel economy. Let me tell you a little about the average car and then I will describe some options and ask you to choose the one you are most likely to purchase. I will read all the options first, then will summarize them and ask you to pick one.

The average car accelerates from 0 to 60 miles per hour in about 10.5 seconds and achieves an EPA fuel economy rating of 27.3 miles per gallon. Your options are:

1. The average car.

2. A car that is 10 percent lighter with the same acceleration performance but a 2 miles per gallon better fuel economy, saving about $\$ 4$ per month in fuel.

3. A car that is 10 percent lighter with the same fuel economy but 1.5 seconds quicker acceleration time.

4. A car that is 10 percent heavier with the same fuel economy but about 1.5 seconds slower acceleration time.

5. A car that is 10 percent heavier with the same acceleration performance but a 2 miles per gallon lower fuel economy, costing about $\$ 4$ more per month in fuel.

Which of these would you most likely purchase? 
Table 5.2.3. Public Preference Toward Purchase of Cars with Different Weight, Acceleration and Fuel Economy Parameters

\begin{tabular}{lrr}
\hline Car & Number & Percent \\
\hline The average car & 55 & 15 \\
The lighter car with average acceleration and better fuel economy & 102 & 27 \\
$\begin{array}{l}\text { The lighter car with average fuel economy and quicker } \\
\quad \text { acceleration }\end{array}$ & 63 & 17 \\
$\begin{array}{l}\text { The heavier car with average fuel economy and slower } \\
\quad \text { acceleration }\end{array}$ & 76 & 20 \\
$\begin{array}{l}\text { The heavier car with average acceleration and lower fuel } \\
\quad \text { economy }\end{array}$ & 59 & 16 \\
Don't know & 18 & 5 \\
\hline Total & 373 & 100 \\
\hline
\end{tabular}

Source: ORCI for NREL (1999b), Study \#70844, N=373 
Q5.2.4: For your next vehicle purchase, what would motivate you to purchase a more fuelefficient vehicle? (open-ended)

Table 5.2.4. Public Perception of Motivation Required to Purchase a More Fuel Efficient Vehicle

\begin{tabular}{|c|c|c|c|c|c|c|c|c|c|c|c|c|c|c|c|c|c|}
\hline \multirow{3}{*}{\multicolumn{2}{|c|}{$\begin{array}{l}\text { Motivation for Purchase of a More } \\
\text { Fuel-Efficient Vehicle }\end{array}$}} & \multicolumn{2}{|c|}{ Total } & \multicolumn{14}{|c|}{ Type of New Vehicle Most Likely to Buy } \\
\hline & & \multirow{2}{*}{$\mathbf{n}$} & \multirow{2}{*}{$\%$} & \multicolumn{2}{|c|}{ Small Car } & \multicolumn{2}{|c|}{$\begin{array}{l}\text { Midsized } \\
\text { Car }\end{array}$} & \multicolumn{2}{|c|}{$\begin{array}{l}\text { Large } \\
\text { Car }\end{array}$} & \multicolumn{2}{|c|}{$\begin{array}{l}\text { Pickup } \\
\text { Truck }\end{array}$} & \multicolumn{2}{|c|}{$\begin{array}{l}\text { Standard } \\
\text { Van }\end{array}$} & \multicolumn{2}{|c|}{ Minivan } & \multicolumn{2}{|c|}{ SUV } \\
\hline & & & & $\mathbf{n}$ & $\%$ & $\mathbf{n}$ & $\%$ & $\mathbf{n}$ & $\%$ & $\mathbf{n}$ & $\%$ & $\mathbf{n}$ & $\%$ & $\mathbf{n}$ & $\%$ & $\mathbf{n}$ & $\%$ \\
\hline \multirow[t]{6}{*}{ Cost/savings (net) } & & 428 & 45 & 40 & 43 & 143 & 43 & 42 & 45 & 71 & 43 & 10 & 52 & 31 & 39 & 91 & 52 \\
\hline & Sticker price of vehicle & 159 & 17 & 7 & 7 & 55 & 16 & 17 & 18 & 24 & 15 & 4 & 20 & 15 & 19 & 37 & 21 \\
\hline & $\begin{array}{l}\text { Lower cost/saves money (general } \\
\text { mentions) }\end{array}$ & 130 & 14 & 19 & 20 & 43 & 13 & 11 & 12 & 22 & 13 & 4 & 21 & 11 & 14 & 20 & 11 \\
\hline & Lower cost of fuel & 121 & 13 & 13 & 14 & 40 & 12 & 13 & 14 & 21 & 13 & 2 & 10 & 3 & 4 & 29 & 16 \\
\hline & Operating/maintenance costs & 25 & 3 & 1 & 1 & 7 & 2 & 3 & 3 & 6 & 4 & 0 & 0 & 3 & 4 & 4 & 2 \\
\hline & All other cost mentions & 18 & 1 & 3 & 4 & 5 & 1 & 3 & 3 & 1 & 1 & 0 & 0 & 1 & 1 & 5 & 2 \\
\hline \multirow{4}{*}{$\begin{array}{l}\text { Features/perform- } \\
\text { ance (net) }\end{array}$} & & 219 & 23 & 2 & 24 & 77 & 23 & 21 & 22 & 41 & 25 & 4 & 21 & 15 & 19 & 38 & 21 \\
\hline & $\begin{array}{l}\text { Acceptable emissions control/less } \\
\text { pollution }\end{array}$ & 104 & 11 & 9 & 10 & 37 & 11 & 13 & 14 & 20 & 12 & 0 & 0 & 7 & 8 & 18 & 10 \\
\hline & Horsepower/speed & 33 & 3 & 1 & 1 & 8 & 2 & 3 & 3 & 13 & 8 & 0 & 0 & 3 & 4 & 5 & 3 \\
\hline & $\begin{array}{l}\text { Other features/styles/options } \\
\text { offered }\end{array}$ & 99 & 11 & 16 & 16 & 41 & 14 & 7 & 7 & 14 & 9 & 5 & 26 & 8 & 10 & 17 & 11 \\
\hline $\begin{array}{l}\text { Gas mileage/fuel } \\
\text { efficiency }\end{array}$ & & 167 & 17 & 16 & 17 & 61 & 18 & 16 & 17 & 33 & 20 & 1 & 7 & 16 & 21 & 24 & 14 \\
\hline Fuel & Availability of type of fuel needed & 46 & 5 & 2 & 2 & 13 & 4 & 5 & 5 & 6 & 4 & 1 & 5 & 5 & 6 & 13 & 8 \\
\hline Other & & 105 & 10 & 14 & 15 & 36 & 10 & 12 & 12 & 20 & 13 & 1 & 4 & 10 & 11 & 15 & 8 \\
\hline $\begin{array}{r}\text { Nothing/not } \\
\text { interested }\end{array}$ & & 74 & 8 & 6 & 6 & 27 & 8 & 8 & 8 & 15 & 9 & 2 & 13 & 5 & 6 & 11 & 6 \\
\hline Don't know & & 87 & 9 & 8 & 9 & 28 & 9 & 9 & 9 & 13 & 8 & 3 & 14 & 10 & 12 & 16 & 9 \\
\hline
\end{tabular}

Source: ORCI for NREL (1998a), Study \#707089, N=961 
Q5.2.5: How much more would you be willing to pay for the vehicle that gets ten percent better fuel economy than for the vehicle you currently drive? (open-ended)

Table 5.2.5. Additional Amount the Public is Willing to Pay for a Vehicle with a 10 Percent Increase in Fuel Economy

\begin{tabular}{lrr}
\hline Dollar Amount & Number & Percent \\
\hline Less than $\$ 500$ & 13 & 7 \\
$\$ 500-\$ 1,000$ & 27 & 15 \\
$\$ 1,001-\$ 2,500$ & 31 & 17 \\
$\$ 2,501-\$ 5,000$ & 26 & 15 \\
More than $\$ 5,000$ & 10 & 5 \\
None & 33 & 18 \\
Don't know & 41 & 23 \\
\hline Total & 181 & 100 \\
\hline Mean' (including none) & 2,143 & \\
\hline Mean' (excluding none) & 2,799 & \\
\hline
\end{tabular}

Source: ORCI for NREL (2001b), Study \#710449, N=180.

${ }^{1}$ In this report, calculation of means, medians and standard deviations are based on raw numbers. "Don't know" responses are not part of the calculations.

Q5.2.6: Suppose you have decided to buy a new vehicle and have a choice of an optional engine that requires a new fuel that costs the same as gasoline and is just as good as gasoline.

Version A: The optional engine costs the same as the conventional one but gets 50 percent more miles per gallon. However, the fuel it requires is sold only at 1 in 10 stations. Which would you most likely buy?

Version B: The optional engine costs the same as the conventional one but gets 50 percent more miles per gallon. However, the fuel it requires is sold only at 1 in 5 stations. Which would you most likely buy?

Version C: The optional engine costs the same as the conventional one but gets 50 percent more miles per gallon. However, the fuel it requires is sold only at 1 in 3 stations. Which would you most likely buy? 
Table 5.2.6. Public Preference Toward Purchasing a More Fuel Efficient Engine with Different Fuel Availability Options

\begin{tabular}{|c|c|c|c|c|c|c|}
\hline \multirow[t]{2}{*}{ Fuel Availability Options } & \multicolumn{2}{|c|}{$\begin{array}{c}\text { The } \\
\text { Conventional } \\
\text { Engine }\end{array}$} & \multicolumn{2}{|c|}{$\begin{array}{c}\text { The Optional } \\
\text { Engine }\end{array}$} & \multicolumn{2}{|c|}{$\begin{array}{c}\text { Don't } \\
\text { Know/Refused }\end{array}$} \\
\hline & $\mathbf{n}$ & $\%$ & $\mathbf{n}$ & $\%$ & $\mathbf{n}$ & $\%$ \\
\hline $\begin{array}{l}\text { The optional engine costs the same as } \\
\text { the conventional one, but gets } \\
50 \% \text { more miles per gallon. } \\
\text { However, the fuel it requires is } \\
\text { sold only at } 1 \text { in } 10 \text { stations. }\end{array}$ & 74 & 66 & 33 & 30 & 4 & 4 \\
\hline $\begin{array}{l}\text { The optional engine costs the same as } \\
\text { the conventional one, but gets } \\
50 \% \text { more miles per gallon. } \\
\text { However, the fuel it requires is } \\
\text { sold only at } 1 \text { in } 5 \text { stations. }\end{array}$ & 69 & 62 & 40 & 36 & 3 & 3 \\
\hline $\begin{array}{l}\text { The optional engine costs the same as } \\
\text { the conventional one, but gets } \\
50 \% \text { more miles per gallon. } \\
\text { However, the fuel it requires is } \\
\text { sold only at } 1 \text { in } 3 \text { stations. }\end{array}$ & 48 & 43 & 59 & 53 & 4 & 4 \\
\hline
\end{tabular}

Source: ORCI for NREL (2000d), Study \#70920, N=111.

Q5.2.7: Have you ever seen a copy of the Government's Fuel Economy Guide in the showroom, on the Internet, or anywhere else?

Table 5.2.7. Site of Review of the Government's Fuel Economy Guide

\begin{tabular}{lcrr}
\hline Place & Number & Percent \\
\hline Any (net) & & 222 & 22 \\
& In a showroom & 163 & 16 \\
& On the Internet & 24 & 2 \\
& Anywhere else & 64 & 6 \\
Don't know/none of these & & 778 & 78 \\
\hline Total & & 1,000 & 100 \\
\hline
\end{tabular}

Source: ORCI for NREL (1999a), Study \#70809, N=1,000. 


\subsection{VEHICLE OWNERS' DECISIONS ABOUT ADVANCED TECHNOLOGY VEHICLES}

\section{Hybrid Electric Vehicles}

Currently two hybrid electric vehicles are available in the United States: the Toyota Prius, roughly the size of a Corolla, and the Honda Insight, a two-seat vehicle about the size of a Civic. The Insight has Environmental Protection Agency ratings of 61 and 68 miles per gallon in city and highway driving, respectively. The Prius is rated at 52 miles per gallon in the city and 45 on the highway. Both cars can achieve speeds of more than 100 miles per hour (The Washington Post, 2001).

According to surveys, American drivers are aware of hybrid electric vehicles (Tables 5.3.1, 5.3.2). But, a majority of them could not name or did not know any of the hybrid electric vehicles (Tables 5.3.3, 5.3.4). In addition, when given a choice between a conventional vehicle and a hybrid vehicle that has the same total range on a single tank of fuel and battery charge, but can travel 50 percent more miles on a gallon of fuel than a conventional vehicle, more Americans would purchase the conventional vehicle than the hybrid one (Table 5.3.5).

Surveys reveal that U.S. drivers tend to have heard more about a Toyota hybrid than about a Honda one (Table 5.3.1). Nonetheless, when asked to provide specific names of hybrid electric vehicles, U.S. drivers tend to mention the name of Honda more frequently than the name of Toyota (Tables 5.3.3, 5.3.4).

According to Honda, buyers of hybrid electric vehicles tend to be technology enthusiasts who want to be the first in their neighborhood to get the car. They are also more likely to be environmentalists who want cars that conserve gasoline and pollute less. In addition, they tend to be young people who just think it is a fun car. Toyota's profile of a Prius buyer is different. Seventy-one percent of Prius buyers are men. Buyers have an average age of 53, a college education and a median income of $\$ 85,900$ a year (The Washington Post, 2001).

Q5.3.1: There are some cars in the U.S. market today that have advanced hybrid-electric powertrains that combine a small electric motor and a small gasoline engine to achieve a higher fuel economy than similar cars. How much have you heard about this technology: a great deal, some, very little, or nothing?

Table 5.3.1. Amount of Information Heard Pertaining to Advanced Hybrid-Electric Powertrains

\begin{tabular}{lcc}
\hline & August 3, 2000 & November 6, 2001 \\
\hline A Great Deal & $13 \%$ & $10 \%$ \\
Some & $33 \%$ & $33 \%$ \\
Very Little & $34 \%$ & $30 \%$ \\
Nothing & $20 \%$ & $26 \%$ \\
Don't Know & $0 \%$ & $2 \%$ \\
\hline
\end{tabular}


Q5.3.2: $\quad$ Please name one of these hybrid-electric cars if you can. (open-ended)

Table 5.3.2. Names of Advanced Hybrid-Electric Vehicles

Known by the Public

\begin{tabular}{lcc}
\hline & August 3, 2000 & November 6, 2001 \\
\hline Any & $36 \%$ & $44 \%$ \\
Honda & $15 \%$ & $24 \%$ \\
Insight & $1 \%$ & $2 \%$ \\
Toyota & $4 \%$ & $11 \%$ \\
Prius & $1 \%$ & $2 \%$ \\
Other & $14 \%$ & $6 \%$ \\
Don't Know & $64 \%$ & $56 \%$ \\
\hline
\end{tabular}

\section{Diesel Vehicles}

In 1997, one in five U.S. drivers would consider buying a diesel engine that got 40 percent better fuel economy and cost $\$ 1,500$ additional (Table 5.3.6). In 1998, on average, Americans said they would be willing to pay $\$ 837$ extra for a diesel engine that gets 30 miles per gallon compared to a gasoline engine that gets 20 miles per gallon ${ }^{3}$ (Table 5.3.7). In 2001, slightly more than one in four drivers would rather purchase a new diesel vehicle that gets 40 percent more miles per gallon but costs additional $\$ 2,000$ than a gasoline one ${ }^{3}$ (Table 5.3.8).

When asked about reasons for choosing a diesel over a gasoline vehicle, almost one in two U.S. adults mentioned fuel economy and the advantage of getting 40 percent more miles per gallon, in particular (Table 5.3.9). Those who preferred a gasoline to a diesel vehicle referred to environmental concerns, such as diesel engines being loud or noisy and having an odor or smell. A few American drivers mentioned engine problems including difficult starting in winter and slow acceleration as a reason for rejecting the diesel vehicle. Some reported negative experiences with diesel (Table 5.3.10).

\footnotetext{
${ }^{3}$ Gasoline and diesel engines were defined as equally clean, dependable, powerful, odorless, and smooth running.
} 
Q5.3.3: Would you consider buying a diesel engine version that got 40 percent better fuel economy and costs an additional $\$ 1,500$ ?

Table 5.3.3. Public's Willingness to Consider the Purchase of a Diesel Engine With a 40 Percent Increase in Fuel Economy and Additional Costs of \$1,500

\begin{tabular}{lc}
\hline Considerations of Diesel Engine Options & Percent \\
\hline Would consider buying a diesel engine version that got $40 \%$ better fuel & 21 \\
economy and costs an additional $\$ 1,500$. & \\
Would not consider buying a diesel engine version that got $40 \%$ better & 75 \\
fuel economy and costs an additional $\$ 1,500$. & \\
$\begin{array}{l}\text { Don't know } \\
\text { Total }\end{array}$ & 4 \\
\hline
\end{tabular}

Source: ORCI for NREL (1997, Study \#70627), N=1010 
Q5.3.4: If you had a choice between two engines for your next vehicle, both engines equally clean, powerful, odorless, and smooth running, one using gasoline and getting 20 miles per gallon, and one using diesel fuel and getting 30 miles per gallon, how much extra would you be willing to pay for the diesel one? (open-ended)

Table 5.3.4. Additional Amount the Public is Willing to Pay for a Diesel Engine Getting 30 Miles per Gallon Compared to a Gasoline Engine Getting 20 Miles per Gallon

\begin{tabular}{|c|c|c|c|c|c|c|c|c|c|c|c|c|c|c|c|c|}
\hline \multirow{3}{*}{ Dollar Amount } & \multicolumn{6}{|c|}{ Total } & \multicolumn{10}{|c|}{ Type of New Vehicle Most Likely to Buy } \\
\hline & \multirow{2}{*}{$\mathbf{n}$} & \multirow{2}{*}{$\%$} & \multicolumn{2}{|c|}{ Small Car } & \multicolumn{2}{|c|}{$\begin{array}{l}\text { Midsized } \\
\text { Car }\end{array}$} & \multicolumn{2}{|c|}{ Large Car } & \multicolumn{2}{|c|}{$\begin{array}{c}\text { Pickup } \\
\text { Truck }\end{array}$} & \multicolumn{2}{|c|}{$\begin{array}{l}\text { Standard } \\
\text { Van }\end{array}$} & \multicolumn{2}{|c|}{ Minivan } & \multicolumn{2}{|c|}{ SUV } \\
\hline & & & $\mathbf{n}$ & $\%$ & $\mathbf{n}$ & $\%$ & $\mathbf{n}$ & $\%$ & $\mathbf{n}$ & $\%$ & $\mathbf{n}$ & $\%$ & $\mathbf{n}$ & $\%$ & $\mathbf{n}$ & $\%$ \\
\hline$\$ 500$ or less & 63 & 7 & 9 & 10 & 16 & 5 & 3 & 3 & 15 & 9 & 1 & 4 & 9 & 12 & 11 & 6 \\
\hline$\$ 501-\$ 1,000$ & 79 & 8 & 11 & 12 & 24 & 7 & 2 & 3 & 18 & 11 & 1 & 6 & 10 & 13 & 11 & 6 \\
\hline$\$ 1,001-\$ 2,000$ & 81 & 8 & 7 & 7 & 29 & 9 & 9 & 10 & 12 & 7 & 1 & 6 & 7 & 8 & 16 & 9 \\
\hline$\$ 2,001-\$ 5,000$ & 71 & 7 & 5 & 6 & 16 & 5 & 9 & 9 & 22 & 14 & 1 & 6 & 3 & 3 & 16 & 9 \\
\hline More than $\$ 5,000$ & 20 & 2 & 1 & 2 & 8 & 2 & 0 & 0 & 4 & 3 & 0 & 0 & 1 & 2 & 5 & 3 \\
\hline None & 533 & 55 & 48 & 51 & 197 & 59 & 59 & 62 & 76 & 47 & 14 & 73 & 41 & 52 & 96 & 54 \\
\hline Don't know & 114 & 12 & 11 & 12 & 43 & 13 & 12 & 13 & 16 & 10 & 1 & 5 & 8 & 10 & 22 & 13 \\
\hline $\begin{array}{l}\text { Mean }^{1} \text { (including } \\
\text { none) }\end{array}$ & 837 & & 723 & & 730 & & 707 & & 1,116 & & 507 & & 692 & & 1,106 & \\
\hline $\begin{array}{l}\text { Mean }^{1} \text { (excluding } \\
\text { none) }\end{array}$ & 2,255 & & 1,740 & & 2,273 & & 2,507 & & 2,299 & & 2,264 & & 1,635 & & 2,688 & \\
\hline
\end{tabular}

Source: ORCI for NREL (1998a), Study \#707089, N=961.

${ }^{1}$ In this report, calculation of means, medians and standard deviations are based on raw numbers. "Don't know" responses are not part of the calculations. 
Q5.3.5: Assume that a new vehicle you want to buy has two engine options that are equally clean, dependable, powerful, odorless, and smooth running. One uses gasoline and the other uses diesel fuel and gets 40 percent more miles per gallon but costs $\$ 2,000$ more. Which engine option would you buy?

Table 5.3.5. Purchase Preference Between Diesel and Gasoline Vehicles by Vehicle Type

\begin{tabular}{|c|c|c|c|c|c|c|c|c|c|c|c|c|c|c|}
\hline \multirow{3}{*}{ Vehicles } & \multirow{2}{*}{\multicolumn{2}{|c|}{ Total }} & \multicolumn{10}{|c|}{ Vehicle Type } & \multirow{2}{*}{\multicolumn{2}{|c|}{$\begin{array}{l}\text { Don't } \\
\text { Know } \\
\text { Which } \\
\text { Type of } \\
\text { Vehicle } \\
\text { to Buy }\end{array}$}} \\
\hline & & & \multicolumn{2}{|c|}{ Small Car } & \multicolumn{2}{|c|}{ Large Car } & \multicolumn{2}{|c|}{ Minivan } & \multicolumn{2}{|c|}{ SUV } & \multicolumn{2}{|c|}{ Pickup/Van } & & \\
\hline & $\mathbf{n}$ & $\%$ & $\mathbf{n}$ & $\%$ & $\mathbf{n}$ & $\%$ & $\mathbf{n}$ & $\%$ & $\mathbf{n}$ & $\%$ & $\mathbf{n}$ & $\%$ & $\mathbf{n}$ & $\%$ \\
\hline Gasoline & 703 & 71 & 178 & 75 & 186 & 81 & 73 & 71 & 126 & 62 & 124 & 65 & 15 & 60 \\
\hline Diesel & 266 & 27 & 50 & 21 & 41 & 18 & 28 & 27 & 75 & 37 & 65 & 34 & 7 & 28 \\
\hline Don't know & 20 & 2 & 9 & 4 & 3 & 1 & 2 & 2 & 2 & 1 & 1 & 1 & 3 & 12 \\
\hline Total & 989 & 100 & 237 & 100 & 230 & 100 & 103 & 100 & 203 & 100 & 190 & 100 & 25 & 100 \\
\hline
\end{tabular}

Source: ORCI for NREL (2001c), Study \#710288, N=989. 
If chose diesel, ask:

Q5.3.6: Why did you choose the diesel option? (open-ended)

Table 5.3.6. Reasons for Choosing a Diesel Option

\begin{tabular}{|c|c|c|c|}
\hline \multirow{2}{*}{ Reasons } & & \multicolumn{2}{|c|}{ Total } \\
\hline & & $\mathbf{n}$ & $\%$ \\
\hline \multirow[t]{3}{*}{ Fuel economy (net) } & & 121 & 46 \\
\hline & Better gas mileage/fuel economy & 100 & 38 \\
\hline & $\begin{array}{l}40 \% \text { better mileage/miles per } \\
\text { gallon }\end{array}$ & 22 & 8 \\
\hline \multirow[t]{4}{*}{ Cost (net) } & & 90 & 34 \\
\hline & $\begin{array}{l}\text { Saves money/pays for itself over } \\
\text { time/in the long run }\end{array}$ & 45 & 17 \\
\hline & $\begin{array}{l}\text { Less expensive/cheaper than } \\
\text { gasoline }\end{array}$ & 32 & 12 \\
\hline & Economy/economical & 18 & 7 \\
\hline \multirow[t]{3}{*}{ Dependability (net) } & & 33 & 12 \\
\hline & Diesel engine lasts longer & 19 & 7 \\
\hline & $\begin{array}{l}\text { Diesel engine more } \\
\text { reliable/dependable }\end{array}$ & 18 & 7 \\
\hline \multirow[t]{3}{*}{ Environmental (net) } & & 26 & 10 \\
\hline & Cleaner/burns cleaner & 18 & 7 \\
\hline & All other environmental mentions & 9 & 3 \\
\hline $\begin{array}{l}\text { I have/drive vehicle with diesel } \\
\text { engine }\end{array}$ & & 11 & 4 \\
\hline More power/horsepower & & 8 & 3 \\
\hline $\begin{array}{l}\text { Previous positive experience/ } \\
\text { satisfied with diesel }\end{array}$ & & 7 & 3 \\
\hline Other /don't know & & 26 & 10 \\
\hline
\end{tabular}

Source: ORCI for NREL (2001c), Study \#710288, N=266. 
If did not choose diesel, ask:

Q5.3.7: Why did you reject the diesel option? (open-ended)

Table 5.3.7. Reasons for Rejecting a Diesel Option

\begin{tabular}{|c|c|c|c|}
\hline \multirow{2}{*}{ Reasons } & & \multicolumn{2}{|c|}{ Total } \\
\hline & & $\mathbf{n}$ & $\%$ \\
\hline \multirow[t]{5}{*}{ Environmental (net) } & & 282 & 39 \\
\hline & Loud/noisy/too much noise & 140 & 19 \\
\hline & Odor/smell/stink & 119 & 16 \\
\hline & Pollutes the air & 32 & 4 \\
\hline & $\begin{array}{l}\text { All other environmental } \\
\text { mentions }\end{array}$ & 53 & 8 \\
\hline \multirow[t]{4}{*}{ Cost (net) } & & 135 & 19 \\
\hline & Cost /expense (unspecified) & 71 & 10 \\
\hline & The initial cost $/ \$ 2000$ more & 34 & 5 \\
\hline & All other cost mentions & 37 & 5 \\
\hline Lack of fuel availability & & 123 & 17 \\
\hline $\begin{array}{l}\text { Don't know enough/know nothing } \\
\text { about it/never owned one }\end{array}$ & & 78 & 11 \\
\hline \multirow[t]{3}{*}{ Engine problems (net) } & & 57 & 8 \\
\hline & Difficult to start in winter & 37 & 5 \\
\hline & $\begin{array}{l}\text { All other engine problems } \\
\text { mentions }\end{array}$ & 21 & 3 \\
\hline $\begin{array}{l}\text { Just don't like diesel/husband } \\
\text { doesn't like diesel }\end{array}$ & & 48 & 7 \\
\hline $\begin{array}{l}\text { Prefer/used to/satisfied with } \\
\text { gasoline }\end{array}$ & & 34 & 5 \\
\hline Negative experience & $\begin{array}{l}\text { Previous negative } \\
\text { experience and Heard of } \\
\text { others' bad experiences }\end{array}$ & 28 & $\overline{4}$ \\
\hline Difficult to maintain/repair & & 22 & 3 \\
\hline Other & & 28 & 4 \\
\hline Don't know & & 28 & 4 \\
\hline
\end{tabular}

Source: ORCI for NREL (2001c), Study \#710288, N=723. 


\section{REFERENCES}

ABC News/Washington Post poll. (2001). May 31-June 3.

Birky et al. (2001). Future U.S. Highway Energy Use: A Fifty Year Perspective, draft, February.

Gallup Poll. (2001a). June 28-July 1.

Gallup Poll. (2001b). May 7-9.

Gallup Poll. (2001c). March 5-7.

Gallup Poll. (2000a). June 22-25.

Gallup Poll. (2000b). May 23-24.

Gallup Poll. (2000c). March 30-April 2.

Gallup Poll. (2000d). March 10-12.

IPSOS-REID Inc. poll. (2001). November 14.

NBC News/Wall Street Journal. (2001). June 23-25.

(ORCI for NREL) Opinion Research Corporation International for National Renewable Energy Laboratory. 2001a. U.S. Dependence On Imported Oil, Study \#710148, April 5.

(ORCI for NREL) Opinion Research Corporation International for National Renewable Energy Laboratory. 2001b. Fuel Economy, Study \#710449, November 2.

(ORCI for NREL) Opinion Research Corporation International for National Renewable Energy Laboratory. (2001c). Purchasing A New Car-Gasoline Versus Diesel, Study \#710288, July 12.

(ORCI for NREL) Opinion Research Corporation International for National Renewable Energy Laboratory. 2000a. Vehicles And Fuel, Study \#709318, August 3.

(ORCI for NREL) Opinion Research Corporation International for National Renewable Energy Laboratory. (2000b). Fuel For Use In Personal Vehicles, Study \#709489, December 1.

(ORCI for NREL) Opinion Research Corporation International for National Renewable Energy Laboratory. (2000c). Vehicles, Study \#709089, February 24.

(ORCI for NREL) Opinion Research Corporation International for National Renewable Energy Laboratory. (2000d). Conventional And Optional Engines, Study \#70920, May 18.

(ORCI for NREL) Opinion Research Corporation International for National Renewable Energy Laboratory. 1999a. New Light Vehicles And Fuel. Closed-End Tabulations, Study \#70809, February 25.

(ORCI for NREL) Opinion Research Corporation International for National Renewable Energy Laboratory. (1999b). Choosing A New Vehicle, Study \#70844, October 28.

(ORCI for NREL) Opinion Research Corporation International for National Renewable Energy Laboratory. (1998a). New Vehicle Purchases, Study \#707089, February 19.

(ORCI for NREL) Opinion Research Corporation International for National Renewable Energy Laboratory. (1998b). Gasoline/ Diesel Fuel Replacements, Study \#707349, August 20. 
(ORCI for NREL) Opinion Research Corporation International for National Renewable Energy Laboratory. (1997). Diesel, Study \#70627, July 7.

Pew Research Center for the People and the Press poll. (1997). November 13-17.

(PIPA) Program on International Policy Attitudes. 2000. Americans On Global Warming Treaty, February 4.

(PIPA) Program on International Policy Attitudes poll. (1998a). February-April.

(PIPA) Program on International Policy Attitudes poll. (1998b). October 22-27.

Research/Strategy/Management, Inc. (2001). U.S. Dependence on Oil Imports, , August 5.

Research/Strategy/Management, Inc. (1998). America Speaks Out On Energy: Foreign Oil Dependency, October 21.

Sustainable Energy Budget Coalition. (1996). America Speaks Out On Energy: A Survey of Public Attitudes on Sustainable Energy Issues, January.

(U.S. DOE/EIA) U.S. Department of Energy, Energy Information Administration. (2001a). Monthly Energy Review, November.

(U.S. DOE/EIA) U.S. Department of Energy, Energy Information Administration. (2001b). Emissions of Greenhouse Gases in the United States 2000, DOE/ EIA-0573 (2000), Washington, DC, November.

(U.S. DOE/EIA) U.S. Department of Energy, Energy Information Administration. (2001c). International Petroleum Monthly, October.

Washington Post, The. (2001). "Hybrid Cars Draw Waiting List of Buyers," May 3.

World Wildlife Fund National Survey, World Wildlife Fund, August 15, 1997. 